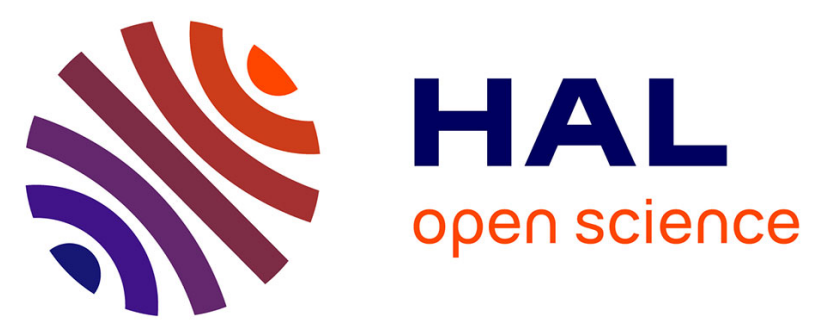

\title{
Prediction of apparent properties with uncertain material parameters using high-order fictitious domain methods and PGD model reduction
}

Grégory Legrain, Mathilde Chevreuil, Naoki Takano

\section{To cite this version:}

Grégory Legrain, Mathilde Chevreuil, Naoki Takano. Prediction of apparent properties with uncertain material parameters using high-order fictitious domain methods and PGD model reduction. International Journal for Numerical Methods in Engineering, 2016, 10.1002/nme.5289 . hal-01303388

\section{HAL Id: hal-01303388 \\ https://hal.science/hal-01303388}

Submitted on 18 Apr 2016

HAL is a multi-disciplinary open access archive for the deposit and dissemination of scientific research documents, whether they are published or not. The documents may come from teaching and research institutions in France or abroad, or from public or private research centers.
L'archive ouverte pluridisciplinaire HAL, est destinée au dépôt et à la diffusion de documents scientifiques de niveau recherche, publiés ou non, émanant des établissements d'enseignement et de recherche français ou étrangers, des laboratoires publics ou privés.

\section{(ㅇ)(1) $\$$}

Distributed under a Creative Commons Attribution - NonCommercial - NoDerivatives 44.0 


\section{Gem

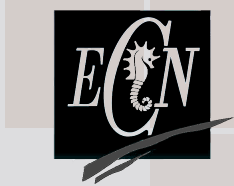

Centrale

Nantes

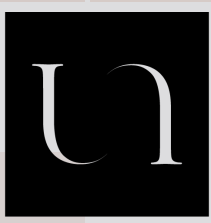

UNIVERSITÉ DE NANTES

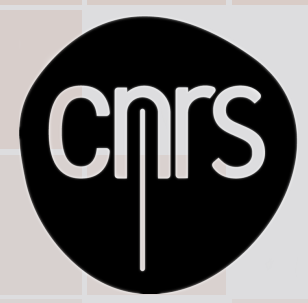

Prediction of apparent properties with uncertain material parameters using high-order fictitious domain methods and PGD model reduction

G.Legrain, M. Chevreuil and N. Takano

\section{GeM Institute}

GeM Institute UMR CNRS 6183

Université Bretagne Loire

École Centrale de Nantes / Université de Nantes / CNRS,

1 Rue de la Noë, BP92101, 44321 Nantes, France. 


\title{
Prediction of apparent properties with uncertain material parameters using high-order fictitious domain methods and PGD model reduction
}

\author{
G.Legrain ${ }^{11}$, M. Chevreuil ${ }^{2}$ and N. Takano ${ }^{3}$ \\ ${ }^{1}$ GeM Institute, UMR CNRS 6183, École Centrale de Nantes, Université de Nantes, France \\ ${ }^{2}$ GeM Institute, UMR CNRS 6183, Université de Nantes, École Centrale de Nantes, France \\ ${ }^{3}$ Department of Mechanical Engineering, Keio University, 3-14-1 Hiyoshi, Yokohama, 223-8522, Japan
}

\begin{abstract}
SUMMARY
This contribution presents a numerical strategy to evaluate the effective properties of imagebased microstructures in the case of random material properties. The method relies on three points: (i) a high-order fictitious domain method; (ii) an accurate spectral stochastic model and (iii) an efficient model reduction method based on the Proper Generalized Decomposition in order to decrease the computational cost introduced by the stochastic model. A feedback procedure is proposed for an automatic estimation of the random effective properties with a given confidence. Numerical verifications highlight the convergence properties of the method for both deterministic and stochastic models. The method is finally applied to a real 3D bone microstructure where the empirical probability density function of the effective behaviour could be obtained.
\end{abstract}

KEY WORDS: fictitious domain method; homogenization; high-order; stochastic; proper generalized decomposition

\section{Introduction}

During the past few years, image-based analysis has been the object of a large interest for a wide range of applications. In particular, a large number of studies have been conducted in the domain of biomechanics since the 90's [1,2] for building patient specific models of bones for osteoporosis treatments. The finite element predictions are used to decide whether surgical interventions are needed, but also to understand the relationship between bone morphology and load transfer. Besides osteoporosis applications, patient specific finite element models have also been considered for the optimization and the design of orthopaedic implants (see e.g. [3, 4]). These techniques have also been widely applied in material science $[5,6]$ to understand the relationship between

\footnotetext{
${ }^{1}$ Correspondence to: G. Legrain. E-Mail: gregory.legrain@ec-nantes.fr
} 
the morphology and the mechanical properties of the materials. Two main families of methods have been designed for building finite element meshes from geometrical data (CT scans, microscopy). Images being represented as a multidimensional array of pixels (voxels in 3D), voxel-based finite element approaches $[1,7,8,9,10]$ can directly translate geometrical acquisitions into numerical models by simply associating a quadrangular (resp. hexahedral) element to every pixel (resp. voxel). In this case, material properties can be assigned based on a segmentation, or directly based on the intensity level of the image (provided that a relationship between image intensity and material properties is given). This approach ie almost completely automatic, but leads to large numerical models (directly proportional to the image resolution). In addition, the stair steps approximation of the geometry is known to decrease stress accuracy near boundaries in addition to the convergence of the model [11]. The second family of methods is based on an image segmentation step, followed by surface triangulation and finally volume meshing $[12,13,14]$. This method allows the use of non-homogeneous (user-defined) mesh density, and thus improve the efficiency of the numerical model. However, mesh generation of complex micro-structures is still a challenging task, especially if distorted elements have to be avoided. To answer the issues raised by these two approaches, alternative methods have emerged recently with the objective to simplify the interaction between geometry and numerical computations. One can cite for example the Finite Cell method from Rank, Düster and co-workers [15, 16, 17, 18], and the eXtended Finite Element Method (X-FEM in the following) [19, 20, 21, 22, 23, 24, 25, 26, 27, 28, 29, 30]. The Finite Cell method is a high-order fictitious domain method which has proved to be very efficient thanks to the approximation properties of $\mathrm{p}$-fem [31, 32, 33]. The fictitious domain nature of the method makes possible to use meshes that do not conform to the geometry, avoiding the use of cumbersome meshing techniques. Instead, the effort is put on the integration of the weak formulation, as the geometrical error in the solution has to be mastered. The method has been applied in the context of design through analysis [34], but also to image-based analysis in both material science [35, 36] and biomechanics $[37,38]$. The use of the X-FEM in the context of CAD and fictitious domain traces back to the work of Belytschko [39]. The use of the X-FEM in the context of image-based analysis has been further developed in $[11,40]$ where a complete strategy was proposed to build accurate numerical models from 2D or 3D images. The use of an octree data structure allowed to decrease the computational cost, but also required a special treatment for enriched hanging nodes [41]. The method has been extended to higher order approximations in $[42,43]$, and proved to be efficient for image-based analysis. Despite being very accurate, these methods assume the material properties to be deterministic. This is not the case in practice, as a large scattering of the properties was observed in the numerous experimental data that are taken as reference for the material properties [10].These uncertainties can have multiple sources: (i) geometrical uncertainties due to the geometrical acquisition process, the associated image treatments and the geometrical discretization; (ii) material uncertainties due to inter-individual variation in the material properties of the tissues or even intra-individual variations depending on the biological site where the bone is located and (iii) experimental uncertainties due to the processing of the samples, mis-alignments in the experimental devices, and sensors accuracy. In 
this contribution, we will restrict ourselves to material uncertainties alone. This source of uncertainty is of prime importance in the context of patient-specific models, as they allow to model both intra and inter-individual material variations. For patient-specific models, geometrical uncertainties are mainly linked to the resolution of the image which is considered as sufficiently large (see the example in section 4). Usually, Monte-Carlo strategies are considered to model the propagation of uncertainties in homogenized models. The X-FEM is appealing in this context, and was used in [44] and [45] to study the statistics of the effective properties against the distribution and shape of material inclusions. In the context of FEM, [46] proposed statistical methods in order to estimate the size of the representative volume element of random composites and the associated homogenized properties. Mathematical bonds on the statistics of the effective properties were also obtained in $[47,48]$ when the RVE is sub-sampled and in [48] in the case of random micro-structures. So far, all these contribution assumed fixed material properties for the different phases. In Basaruddin et al. [10], both geometrical and material randomness were taken into account by means of perturbation techniques. Wille et al [49] also studied the impact of the variability of the material properties on the mechanical response of femurs. This approach was relying on a simple product of the deterministic response by an identified stochastic function.

The aim of this manuscript is to take into account state of the art stochastic methods for an accurate representation of the stochastic output of the model. The stochastic finite element method will be considered in the following [50, 51]. However, this method is known to require high computational resources. This is why model reduction techniques will be considered so that large stochastic models can be solved accurately. The accuracy of the model will be ensured, not only at the deterministic level, like in [52,38] but for the whole stochastic model through feedback strategies [33] that proved to be efficient in the p-fem context. As stated above, randomness is considered only for the material properties (to model inter-individual variations). In the case where the resolution of the input images is not sufficient, one could take into account the influence of the image treatment following the approach proposed in [10] which would lead to a random levelset.

The manuscript is organized as follows: first, the numerical strategy is introduced, both at the deterministic and stochastic levels. Emphasize is also put on the PGD (Proper Generalized Decomposition) model reduction technique [53, 54, 55] in the context of stochastic analysis. In a second part, the numerical strategy is validated on a $2 \mathrm{D}$ model problem, before being applied to a real 3D cortical bone micro-structure.

\section{Numerical strategy}

The numerical strategy which is proposed in this contribution is based on three ingredients: (i) an efficient and accurate numerical scheme for computing the overall properties of image-based structures, (ii) an accurate stochastic model to take into account the uncertainties in the model and (iii) an efficient model reduction method to decrease the 
computational cost of the stochastic model. These aspects are detailed in the following sections.

\subsection{High order fictitious domain method}

One of the main difficulties for simulating image-based micro-structures concerns the treatment of the amount of geometrical informations produced during the acquisition. Indeed, $1024^{3}$ voxels images are now common: such high-resolution images require large computer resources for the simulations unless tedious geometrical and meshing treatments are used. In this contribution, an alternative path is followed: geometrically non-conforming approaches in the spirit of fictitious domain methods are considered in order to alleviate the meshing burden. The method is illustrated in figure 1 a: The approximation is defined on a regular background grid of simplices or hypercubes which does not conform to the geometry. Elements that are fully in the domaine are integrated with classical quadrature rules, whereas ad hoc strategies have been developed for elements lying partially in the domain. The objective is to integrate accurately the weak formulation on these elements. The Finite Cell method [35, 17, 16, 56, 18] initially used space-tree algorithms and integration points classification (see figure $1 \mathrm{~b}$ ), whereas so-called sub-grid level-sets was advocated in $[42,43]$ (see figure $1 \mathrm{c}$ ) where the geometry is represented using the iso-zero of a level-set function [57]. With respect to space-tree algorithms, this method employs a local tesselation near the interface. More recently, the use of suitable mappings was proposed in order to decrease the integration cost when the interfaces are represented by means of parametric surfaces [58, 59, 60, 61, 62] (see figure $1 \mathrm{~d}$ ). As we are dealing with discrete geometries, we will focus on sub-grid level-sets in the following. The computational strategy proposed in $[40,11]$ is used to convert the images into numerical models using level-sets.

Thanks to this approach, it is possible to treat very complex geometries with an approximation defined on a regular grid. Accurate results with such coarse meshes are obtained thanks to the use of high-order approximations (typically up to $p=10$ in $2 \mathrm{D}$, and $p=8$ in 3D). This method has been proved to be very effective in previous contributions. This stems from the fact that $p$ methods can attain exponential convergence whereas classical $h$ finite elements are limited to algebraic convergence [33].

\subsection{Numerical homogenization}

We are interested in the computation of the overall properties of material micro-structures. The output of the procedure is the effective elasticity tensor $\mathcal{C}$ which relates the macroscopic stress and strain tensors, $\underline{\underline{\Sigma}}$ and $\underline{\underline{E}}$ :

$$
\underline{\underline{\Sigma}}=\mathcal{C} \underline{\underline{E}}
$$




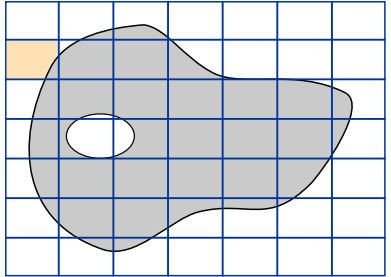

a)

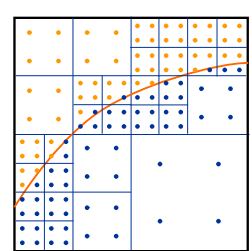

b)

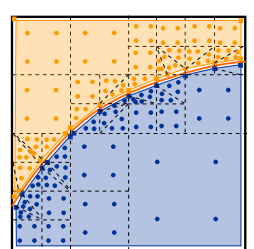

c)

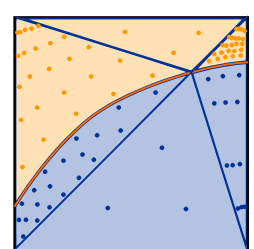

d)

Figure 1: Unfitted approximation of a body, and quadrature points for an element crossed by the interface: b) Finite Cell quad-tree algorithm, c) sub-grid levelset and d) transfinite mapping.

where $\underline{\underline{\underline{\Sigma}}}$ and $\underline{\underline{E}}$ are defined by the following spatial averages:

$$
\begin{aligned}
\underline{\underline{\underline{E}}} & =\langle\underline{\underline{\sigma}}\rangle, \\
\underline{\underline{E}} & =\langle\underline{\underline{\varepsilon}}\rangle, \\
<\bullet> & =\frac{1}{V} \int_{\Omega} \bullet d \Omega
\end{aligned}
$$

where tensors $\underline{\underline{\sigma}}$ and $\underline{\underline{\varepsilon}}$ are the micro stress and strain tensors, $\Omega$ is the spatial domain, and $V$ its volume. The homogenization procedure is based here on kinematic uniform boundary conditions (KUBC), but could also be based on stress uniform boundary conditions (SUBC) or periodic boundary conditions (PBC) [46, 63, 48]:

$$
\mathbf{u}=\underline{\underline{E}} \cdot \mathbf{x} \quad \text { on } \partial \Omega
$$

where $\mathbf{u}$ is the displacement. Using these boundary conditions, equation (3) is automatically verified, whereas the components of $\mathcal{C}$ are obtained by computing eq.(2) for three macroscopic displacement modes associated to macroscopic strain fields $\underline{\underline{E}}_{1}, \underline{\underline{E}}_{2}$ and $\underline{\underline{E}}_{3}$ (2D macroscopic tractions and shear):

$$
\begin{aligned}
& \underline{\underline{E}}_{1}=\left[\begin{array}{ll}
1 & 0 \\
0 & 0
\end{array}\right], \\
& \underline{\underline{E}}_{2}=\left[\begin{array}{ll}
0 & 0 \\
0 & 1
\end{array}\right], \\
& \underline{\underline{E}}_{3}=\left[\begin{array}{cc}
0 & 0.5 \\
0.5 & 0
\end{array}\right] .
\end{aligned}
$$

If a so called energetic extraction is considered, then the components of $\mathcal{C}$ can also be obtained by means of the Hill-Mandel condition:

$$
\mathcal{C}_{i j}=\underline{\underline{E}}_{i} \cdot \mathcal{C} \cdot \underline{\underline{E}}_{j}=a\left(\mathbf{u}_{i}, \mathbf{u}_{j}\right)
$$


where $a(\mathbf{u}, \mathbf{v})=\int_{\Omega} \underline{\underline{\varepsilon}}(\mathbf{u}): \mathcal{K}: \underline{\underline{\varepsilon}}(\mathbf{v}) d \Omega$ is the bilinear form of the weak formulation of the mechanical problem with $\overline{\overline{\mathcal{K}}}$ the fourth order Hooke tensor, and $\mathbf{u}_{i}$ is the microdisplacement associated to $\underline{\underline{E}}_{i}$ in (5). If $\mathbf{u}_{i}^{h, p}$ is the finite element solution of the weak formulation of the unit-cell problem, then the associated finite element effective tensor verifies:

$$
\mathcal{C}_{i j}^{h, p}=\underline{\underline{E}}_{i} \cdot \mathcal{C}^{h, p} \cdot \underline{\underline{E}}_{j}=a\left(\mathbf{u}_{i}^{h, p}, \mathbf{u}_{j}^{h, p}\right)
$$

Following [52], we can write that:

$$
\begin{aligned}
\mathcal{C}_{i j}^{h, p}-\mathcal{C}_{i j} & =a\left(\mathbf{u}_{i}^{h, p}, \mathbf{u}_{j}^{h, p}\right)-a\left(\mathbf{u}_{i}, \mathbf{u}_{j}\right) \\
& =a\left(\mathbf{u}_{i}^{h, p}-\mathbf{u}_{i}, \mathbf{u}_{j}^{h, p}-\mathbf{u}_{j}\right)
\end{aligned}
$$

Using Schwarz' inequality, the expression can be bounded:

$$
\left|\mathcal{C}_{i j}^{h, p}-\mathcal{C}_{i j}\right| \leq a\left(\mathbf{u}_{i}^{h, p}-\mathbf{u}_{i}, \mathbf{u}_{i}^{h, p}-\mathbf{u}_{i}\right)^{1 / 2} a\left(\mathbf{u}_{j}^{h, p}-\mathbf{u}_{j}, \mathbf{u}_{j}^{h, p}-\mathbf{u}_{j}\right)^{1 / 2}
$$

Noting that $a\left(\mathbf{u}_{i}^{h, p}-\mathbf{u}_{i}, \mathbf{u}_{i}^{h, p}-\mathbf{u}_{i}\right)^{1 / 2}$ is the energy norm of the error of micro-problem $i$, we can use classical finite elements error estimates:

$$
\begin{aligned}
\left\|e_{i}\right\|_{E}=a\left(\mathbf{u}_{i}^{h, p}-\mathbf{u}_{i}, \mathbf{u}_{i}^{h, p}-\mathbf{u}_{i}\right)^{1 / 2} & \leq C h^{p} \simeq \tilde{C} N^{-p / d i m} \quad \text { for } h \text { finite elements } \\
& \leq k / \exp \left(\gamma N^{\theta}\right) \quad \text { for } p \text { finite elements, }
\end{aligned}
$$

where $\operatorname{dim}$ is the dimension of the problem, $N$ the number of degrees of freedom and $C, \tilde{C}, k, \beta$ and $\theta$ are problem dependent constants. It can be shown that $\theta>1 / 2$ in $2 \mathrm{D}$ (resp. $1 / 3$ in $3 \mathrm{D}$ ) if the solution is regular. Otherwise, the converge rate remains algebraic, see [33] for the derivation of these estimates. This means that the convergence of the components of effective tensor behaves like the error of the micro-solution:

$$
\begin{aligned}
\left|\mathcal{C}_{i j}^{h, p}-\mathcal{C}_{i j}\right| & \leq C h^{2 p} \simeq \tilde{C} N^{-2 p / \operatorname{dim}} \text { for } h \text { finite elements, } \\
& \leq k / \exp \left(2 \gamma N^{\theta}\right) \quad \text { for } p \text { finite elements. }
\end{aligned}
$$

Note however that $\mathcal{C}_{i j}^{h, p}$ may converge to $\mathcal{C}_{i j}$ from above or below depending on the component. Thus, the absolute value in (16) can be dropped for the diagonal components, but not for the off-diagonal ones. Nevertheless it is still possible to apply the extrapolation estimate proposed in [33] to estimate the error on these quantities of interest. Indeed, [33] shows that $\mathcal{C}_{i j}$ can be estimated from three computations with degrees $p$, $p-1, p-2$ by solving the following nonlinear equation:

$$
\frac{\left(\mathcal{C}_{i j}^{h, p}-\mathcal{C}_{i j}\right)}{\left(\mathcal{C}_{i j}^{h, p-1}-\mathcal{C}_{i j}\right)}=\left[\frac{\left(\mathcal{C}_{i j}^{h, p-1}-\mathcal{C}_{i j}\right)}{\left(\mathcal{C}_{i j}^{h, p-2}-\mathcal{C}_{i j}\right)}\right]^{Q}
$$

with $Q=\frac{\log \left(\frac{N_{p-1}}{N_{p}}\right)}{\log \left(\frac{N_{p-2}}{N_{p-1}}\right)}$. Although less powerful, this approach is easier to implement than goal-oriented error estimators which require 6 (21 in 3D) additional adjoint resolutions to estimate the error. The performances of this adaptive method will be studied in the following sections. 


\subsection{Stochastic Finite Elements}

We are interested in the prediction of the impact of uncertainties on the overall behaviour of materials. This impact is traditionally obtained using sampling techniques, such as Monte Carlo simulations. However, such a strategy is intractable because of the large number of large simulations it requires $\left(O\left(1 / \sqrt{N_{\text {samples }}}\right)\right.$ convergence). Perturbationbased methods $[64,10]$ can be used with good results. However, the accuracy of such methods is satisfactory only for a small range of variation of the input parameters. If not, one has to rely on improved schemes like [65]. We will focus here on a functional point of view for stochastic analysis: random quantities are seen as functions of random parameters that represent basic uncertainties. This vision has led to the development of spectral stochastic methods $[50,66,67,51]$. The main advantage of these methods is the explicit representation of the random response of the model as a function of the basic random parameters. They also allow for fast post-processing of the solution (envelope of the solution, sensitivity with respect to the input parameters,...).

In the following, uncertainties are represented by means of a finite number of random variables $\boldsymbol{\xi}=\left\{\xi_{1}, \xi_{2}, \cdots \xi_{r}\right\}$ which are random parameters of the mechanical problem (Young's modulus and Poisson's ratio in our case). Let $\left(\Theta, \mathcal{B}, P_{\xi}\right)$ be the associated probability space, where $\Theta \in \mathbb{R}^{r}$ is the range of $\boldsymbol{\xi}, \mathcal{B}$ the associated $\sigma$-algebra and $P_{\boldsymbol{\xi}}$ the probability measure of $\boldsymbol{\xi}$. The spatial weak formulation of the mechanical problem is: Find $\mathbf{u}(\mathbf{x}, \boldsymbol{\xi}) \in \mathcal{V}^{\Xi}$ such that

$$
a(\mathbf{u}, \mathbf{v} ; \boldsymbol{\xi})=b(\mathbf{v} ; \boldsymbol{\xi}) \quad \forall \mathbf{v} \in \mathcal{V}_{0}^{\Xi}
$$

where $\mathbf{u}(\mathbf{x}, \boldsymbol{\xi})$ is the unknown random displacement field, $\Omega$ is the spatial domain of interest, and $a$ and $b$ represent respectively the bilinear and linear forms of the problem:

$$
\begin{aligned}
a(\mathbf{u}, \mathbf{v} ; \boldsymbol{\xi}) & =\int_{\Omega} \underline{\underline{\varepsilon}}(\mathbf{u}): \mathcal{K}(\boldsymbol{\xi}): \underline{\underline{\varepsilon}}(\mathbf{v}) d \Omega, \\
b(\mathbf{v} ; \boldsymbol{\xi}) & =\int_{\Omega} \mathbf{v} \cdot \mathbf{b}(\boldsymbol{\xi}) d \Omega+\int_{\Gamma_{N}} \mathbf{v} \cdot \mathbf{T}(\boldsymbol{\xi}) d \Gamma .
\end{aligned}
$$

In these expressions, $\mathcal{K}(\boldsymbol{\xi})$ is the random fourth order Hooke tensor, $\mathbf{b}(\boldsymbol{\xi})$ are the random body forces and $\mathbf{T}(\boldsymbol{\xi})$ are the random prescribed tractions on $\Gamma_{N} \in \partial \Omega$. Under suitable regularity assumptions on the random parameters, the solution of (19) is searched in $L^{2}\left(\Theta, \mathcal{B}, P_{\boldsymbol{\xi}} ; \mathcal{V}\right)$ the space of real value square integrable functions defined on the probability space $\left(\Theta, \mathcal{B}, P_{\boldsymbol{\xi}}\right)$ with values in $\mathcal{V}=H^{1}(\Omega)$. Note that this space can be identified with the tensor product space $\mathcal{V} \otimes \mathcal{S}$, with $\mathcal{S}=L^{2}\left(\Theta, \mathcal{B}, P_{\boldsymbol{\xi}}\right)$ the space of real value second order random variables. A finite element approximation of the solution can then be obtained by introducing a finite dimensional space $\mathcal{V}^{N} \subset \mathcal{V}$ such that:

$$
\mathbf{u}(\mathbf{x}, \boldsymbol{\xi}) \approx \mathbf{u}^{h, p}(\mathbf{x}, \boldsymbol{\xi})=\sum_{\alpha=1}^{N} u^{\alpha}(\boldsymbol{\xi}) \mathbf{N}^{\alpha}(\mathbf{x})
$$

where $\left\{\mathbf{N}^{\alpha}\right\}_{\alpha=1}^{N}$ is the basis of functions of the approximation space $\mathcal{V}^{N}$ and $u^{\alpha}$ are the $N$ random degrees of freedom (dofs). Spectral stochastic methods consist in looking for 
an approximation of the dofs $u^{\alpha}(\boldsymbol{\xi})$ under the expansion:

$$
u^{\alpha}(\boldsymbol{\xi}) \approx \sum_{k=1}^{P} u_{k}^{\alpha} H_{k}(\boldsymbol{\xi})
$$

where $\left\{H_{k}(\boldsymbol{\xi})\right\}_{k=1}^{P}$ is a multidimensional basis of $r$ parameters $\left(\xi_{1}, \cdots \xi_{r}\right)$ of an approximation space $\mathcal{S}^{P} \subset S$ (e.g. the polynomial chaos [50,66], piecewise polynoms, wavelets $[68,69,70])$. The coefficients of the expansion can be determined using the weak stochastic formulation of (19): Find $\mathbf{u}^{h, p}(\mathbf{x}, \boldsymbol{\xi}) \in \mathcal{V}^{N} \otimes \mathcal{S}^{P}$ such that:

$$
A\left(\mathbf{u}^{h, p}, \mathbf{v}\right)=B(\mathbf{v}) \quad \forall \mathbf{v} \in \mathcal{V}^{N} \otimes \mathcal{S}^{P}
$$

where:

$$
\begin{aligned}
A(\mathbf{u}, \mathbf{v}) & =\operatorname{Exp}(a(\mathbf{u}(\boldsymbol{\xi}), \mathbf{v}(\boldsymbol{\xi}) ; \boldsymbol{\xi})), \\
B(\mathbf{v}) & =\operatorname{Exp}(b(\mathbf{v}(\boldsymbol{\xi}) ; \boldsymbol{\xi}))
\end{aligned}
$$

where $\operatorname{Exp}(\bullet)=\int_{\Theta} \bullet d P_{\boldsymbol{\xi}}$ is the mathematical expectation. The corresponding linear system contains $N \times P$ equations, where $N=\operatorname{dim}\left(\mathcal{V}^{N}\right)$ and $P=\operatorname{dim}\left(\mathcal{S}^{P}\right)$ may both be large. This makes stochastic finite elements a computationally intensive method. For large deterministic models like those classically encountered in image-based analysis, such an approach would require large computer resources. This difficulty is also known as the "curse of the dimensionality", which motivates the use of a model reduction method for solving the stochastic equations.

\subsection{PGD spectral model reduction}

The Proper Generalized Decomposition [53, 54, 55] is presented in this section for the resolution of problem $(24)^{2}$. This method was initially called Generalized Spectral Decomposition (GSD) [73] in the stochastic framework. The idea is to exploit the tensor structure of the solution, and look for an optimal separated representation of $\mathbf{u}$ under the form:

$$
\mathbf{u}_{m}(\mathbf{x}, \boldsymbol{\xi})=\sum_{i=1}^{m} \mathbf{w}_{i}(\mathbf{x}) \lambda_{i}(\boldsymbol{\xi})
$$

where $\mathbf{w}_{i}(\mathbf{x}) \in \mathcal{V}^{N}$ are deterministic modes, $\lambda_{i} \in \mathcal{S}^{P}$ are stochastic functions and $m$ is the rank which is supposed to be small. This separated representation is constructed without knowing the solution of (24). More recently, it was extended to the full tensorisation of $\mathcal{S}^{P}$ for independent random variables $\left(\xi_{1}, \cdots, \xi_{r}\right)$ with $\Theta=\Theta_{1} \times \cdots \times \Theta_{r}$ and $d P_{\xi}=$ $d P_{\xi_{1}} \cdots d P_{\xi_{r}}: \mathcal{S}^{P}=\mathcal{S}^{P_{1}} \otimes \mathcal{S}^{P_{2}} \cdots \otimes \mathcal{S}^{P_{r}}$ and:

$$
\mathbf{u}_{m}(\mathbf{x}, \boldsymbol{\xi})=\sum_{i=1}^{m} \mathbf{w}_{i}(\mathbf{x}) \phi_{i}^{1}\left(\xi_{1}\right) \cdots \phi_{i}^{r}\left(\xi_{r}\right) .
$$

\footnotetext{
${ }^{2}$ Note that the method itself has already been applied to the resolution of image-based problems, see for instance [71] and [72]
} 
This full tensorisation will make possible the use of very fine stochastic discretization for each random variable $\xi_{j}$ at low cost. This decomposition can be built a priori by recasting problem (24) as the minimization of the functional $J(\mathbf{v})=\frac{1}{2} A(\mathbf{v}, \mathbf{v})-B(\mathbf{v})$ :

$$
\mathbf{u}(\mathbf{x}, \boldsymbol{\xi})=\arg \min _{\mathbf{v} \in \mathcal{V}^{N} \otimes \mathcal{S}^{P_{1} \otimes \cdots \otimes \mathcal{S}^{P_{r}}}} J(\mathbf{v}) .
$$

The decomposition is built progressively by successive addition of rank-one elements $\mathbf{w}(\mathbf{x}) \phi^{1}\left(\xi_{1}\right) \cdots \phi^{r}\left(\xi_{r}\right)$ to a previously computed decomposition $\mathbf{u}_{m-1}$ as:

$$
\mathbf{u}_{m}=\mathbf{u}_{m-1}+\mathbf{w}(\mathbf{x}) \phi^{1}\left(\xi_{1}\right) \cdots \phi^{r}\left(\xi_{r}\right)
$$

The additional term is obtained by finding the best additional term defined as:

$$
\mathbf{w}(\mathbf{x}) \phi^{1}\left(\xi_{1}\right) \cdots \phi^{r}\left(\xi_{r}\right)=\arg \min _{\substack{\mathbf{y}(\mathbf{x}) \chi^{1}\left(\xi_{1}\right) \cdots \chi^{r}\left(\xi_{r}\right) \in \in \\ \mathcal{V}^{N} \otimes \mathcal{S}^{P} \otimes \cdots \otimes \mathcal{S}^{P r}}} J\left(\mathbf{u}_{m-1}+\mathbf{y}(\mathbf{x}) \chi^{1}\left(\xi_{1}\right) \cdots \chi^{r}\left(\xi_{r}\right)\right)
$$

This nonlinear minimization problem is solved using a simple fixed-point algorithm along each components of $\mathbf{w}(\mathbf{x}) \phi^{1}\left(\xi_{1}\right) \cdots \phi^{r}\left(\xi_{r}\right)$. This fixed point algorithm requires the resolution of $r$ 1D stochastic problems and one 3D deterministic problem at each iteration. Usually, 4 to 5 iterations are sufficient to reach stagnation, which makes this algorithm attractive. In this paper, $r=2$, as the two random variables parametrize respectively Young's modulus and Poisson's ratio. The fixed point algorithm consists in solving alternatively three problems:

1. Finding $\mathbf{w}(\mathbf{x})$ with $\phi^{1}\left(\xi_{1}\right)$ and $\phi^{2}\left(\xi_{2}\right)$ considered as known: this problem has size $\operatorname{dim}\left(\mathcal{V}^{N}\right)$.

2. Finding $\phi^{1}\left(\xi_{1}\right)$ with $\mathbf{w}(\mathbf{x})$ and $\phi^{2}\left(\xi_{2}\right)$ considered as known: this problem has size $\operatorname{dim}\left(\mathcal{S}^{P_{1}}\right)$.

3. Finding $\phi^{2}\left(\xi_{2}\right)$ with $\mathbf{w}(\mathbf{x})$ and $\phi^{1}\left(\xi_{1}\right)$ considered as known: this problem has size $\operatorname{dim}\left(\mathcal{S}^{P_{2}}\right)$.

Thus, a collection of moderate size problems has to be solved $(4 \times m$ problems of size $\operatorname{dim}\left(\mathcal{V}^{N}\right), 4 \times m$ problems of size $P_{1}=\operatorname{dim}\left(\mathcal{S}^{P_{1}}\right)$ and $4 \times m$ problems of size $P_{2}=$ $\operatorname{dim}\left(\mathcal{S}^{P_{2}}\right)$ ), rather than one problem $(24)$ of $\operatorname{size} \operatorname{dim}\left(\mathcal{V}^{N} \otimes \mathcal{S}^{P}\right)=N \times P$. The derivation of these problems is presented in appendix A. Note however that the efficiency of the method relies on the low rank separated representations of the operator $A$ and righthand-side $B$ of the weak formulation, these separated representations are precised in the appendix. It was shown that such an algorithm was very robust and converging very fast when efficient update strategies are considered [73]. In order to improve the efficiency of the method, an adaptive stochastic model could be defined at this level. It is not considered here as the model reduction method makes the cost of the stochastic dimensions negligible compared to the deterministic problem (set of $1 \mathrm{D}$ problems). 


\section{2D numerical verifications}

First, 2D numerical verifications are conducted in order to assess the accuracy of the method and highlight its features for both stochastic and deterministic problems. Consider the porous unit-cell of size $1 \mathrm{~mm} \times 1 \mathrm{~mm}$ presented in figure 2. It is composed of a linear elastic material whose Young's modulus is $1 \mathrm{Mpa}$ and Poisson's ratio 0.3. The geometry of the hole's interface is represented by the iso-zero of a linear level-set function interpolated on an adapted mesh whose level of recursion is 12 (i.e. elements of size $2.410^{-4} \mathrm{~mm}$, see figure 2). Note that the geometrical accuracy is fixed for all the following computations. The approximation of the mechanical fields is defined on regular meshes composed of $n \times n$ linear elements along each side for $h$ computations $(n=2,4, \cdots, 256)$, or alternatively $4 \times 4$ elements along each side for $p$ computations. Let introduce the relative error on the 2-norm:

$$
\epsilon\left(\mathcal{C}^{h, p}, \mathcal{C}\right)=\frac{\left\|\mathcal{C}^{h, p}\right\|_{2}-\|\mathcal{C}\|_{2}}{\|\mathcal{C}\|_{2}}
$$

where $\|\cdot\|_{2}$ denotes the 2-norm. The reference error $\epsilon\left(\mathcal{C}^{h, p}, \mathcal{C}^{\text {ref }}\right)$ is computed with respect to an overkill solution $\mathcal{C}^{\text {ref }}$ of the problem (645 293 quadratic quadrangular elements, 1941643 nodes, 3868233 dofs). We begin with deterministic verifications, then continue with stochastic ones.
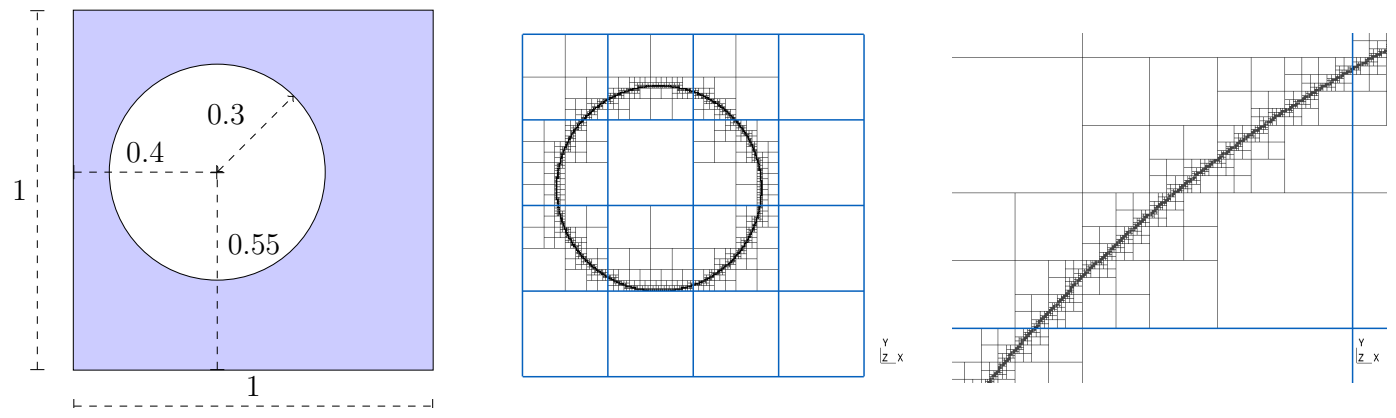

Figure 2: Definition of the unit cell of interest for the 2D verifications.

\section{1. $h$ convergence}

$h$-convergence is first considered in order to study the evolution of the different components of the homogenized tensor $\mathcal{C}$. As stated above, the geometrical accuracy is fixed during the convergence, which contrasts with usual low-order finite elements where geometrical accuracy and mechanical accuracy are linked together. The results of the study are presented in figure 3(a) for the reference error on the tensor $\epsilon\left(\mathcal{C}^{h, p}, \mathcal{C}^{r e f}\right)$ or the component wise reference error $\epsilon\left(\mathcal{C}_{i j}^{h, p}, \mathcal{C}_{i j}^{r e f}\right)$, and 3(b) for the estimated errors $\epsilon\left(\mathcal{C}^{h, p}, \mathcal{C}^{e s t}\right)$ or $\epsilon\left(\mathcal{C}_{i j}^{h, p}, \mathcal{C}_{i j}^{e s t}\right)$ based on the extrapolation estimator presented in section 2.1 where $\mathcal{C}_{i j}^{e s t}$ is 
solution of the nonlinear equation (18). Figure 3(a) shows that all the components of the homogenized tensor converge at the expected algebraic rate $(O(N)$, see section 2.1). The estimated error $\epsilon\left(\mathcal{C}_{i j}^{h, p}, \mathcal{C}_{i j}^{e s t}\right)$ also converges at the expected rate, but the effectivity of the estimator depends on the component of interest (see figures 3(b) and 4). It is very good for the diagonal components of $\mathcal{C}$ (and also $\mathcal{C}_{12}$ ), even for a moderate number of dofs. On the contrary, the estimated error exhibits large oscillations for off-diagonal components. This can be explained by the behaviour of the reference error in figure 3(a): a large number of dofs is required in order to enter the asymptotic range of the convergence. These oscillations prevent the estimator to give reliable results until they stabilize for at least three consecutive computations. This behaviour can be explained as the Galerkin method gives the solution with the lowest error in the energy norm, which is the lowest error in the diagonal terms of $\mathcal{C}$ : Off-diagonal terms are not of primary interest. It is worth noting that in this example the components which exhibit these oscillations have small values (400 times smaller than the other ones). Still, they are not zero (because of the geometry of the problem of interest) and even the absolute error exhibits such oscillations. If rather than working component per component we focus on $\epsilon\left(C^{h, p}, C\right)$ , the error on the 2-norm of $\mathcal{C}$, then the influence of these components is discarded and the estimator gives very reliable results, as depicted in figures 3 and 4 .

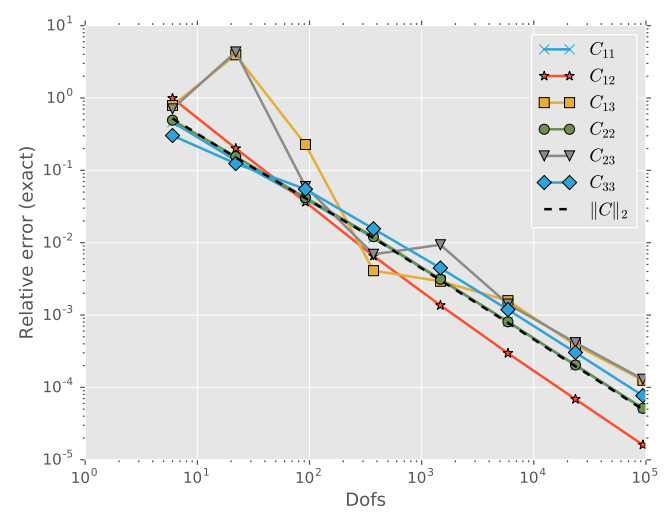

(a) Reference error

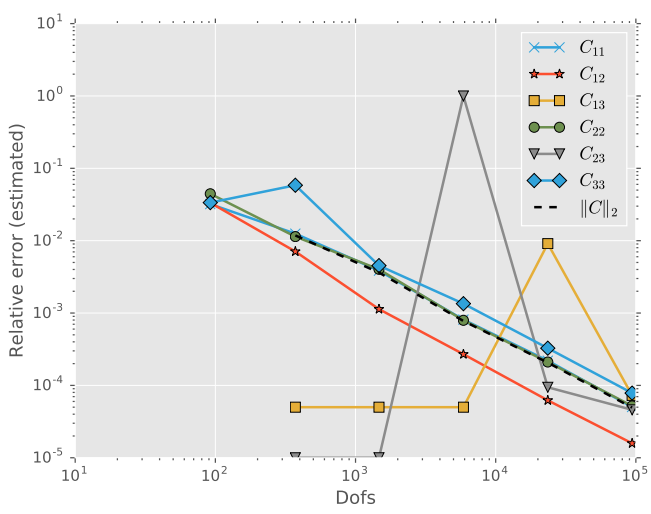

(b) Estimated error

Figure 3: 2D example: (a) reference errors $\epsilon\left(\mathcal{C}^{h, p}, \mathcal{C}^{r e f}\right)$ and $\epsilon\left(\mathcal{C}_{i j}^{h, p}, \mathcal{C}_{i j}^{r e f}\right)$ and (b) estimated errors $\epsilon\left(\mathcal{C}^{h, p}, \mathcal{C}^{e s t}\right)$ and $\epsilon\left(\mathcal{C}_{i j}^{h, p}, \mathcal{C}_{i j}^{e s t}\right)$ of the homogenized tensor $\mathcal{C}$ and its components ( $h$ convergence).

\section{2. $p$ convergence}

The problem is solved by means of $p$ refinement. The computational mesh is highlighted in blue in figure 2), and the spatial polynomial order $p$ ranges from 1 to 7 . The convergence of the components of $\mathcal{C}$ are shown in figure $5(\mathrm{a})$ and (b) for the reference and 


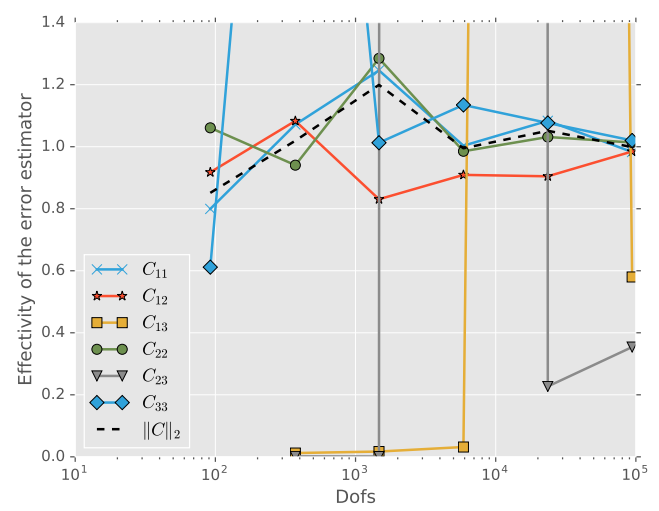

Figure 4: 2D example: Evolution of the effectivity of the estimators $\frac{\epsilon\left(\mathcal{C}_{i j}^{h, p}, \mathcal{C}_{i j}^{e s t}\right)}{\epsilon\left(\mathcal{C}_{i j}^{h, p}, \mathcal{C}_{i j}^{r e f}\right)}$ and $\frac{\epsilon\left(\mathcal{C}^{h, p}, \mathcal{C}^{e s t}\right)}{\epsilon\left(\mathcal{C}^{h, p}, \mathcal{C}^{r e f}\right)}(h$ convergence).

estimated errors. As expected, the convergence is exponential for all the components. This exponential convergence is smoother for the diagonal coefficients, and is oscillatory for $C_{13}$ and $C_{23}$. For the same reasons as in the last section, the estimated error is not able to give reliable results for these two components. Still, working with the 2-norm of $\mathcal{C}$ allows to obtain an accurate estimation of the error of this quantity (see figures 5 and 6). The worse performance of the estimator for $p$-convergence with respect to $h$ convergence which is highlighted in figure 6 may also be related to the small number of dofs for this computation (1400 dofs compared to 100000 dofs for $h$ convergence). If the range of figure 4 is restricted to the same range of dofs for both $h$ and $p$ approximations, then the behaviour is similar but a little better for $h$ convergence. Besides, the error level for $p$-convergence is three orders of magnitude smaller than $h$ computations so that the effectivity becomes very sensitive for $p$ computations, even for this low number of dofs. These numerical experiments also allow us to draw some partial conclusions:

- All the components of the homogenized tensor converge exponentially to their limit value.

- For low number of dofs, the convergence is monotonous only for the diagonal terms of $\mathcal{C}$. This is why the extrapolation error estimator is not robust for the other components. More robust error estimators could be considered, see [74] for a review, or [52, 38] for homogenization applications.

- If the norm of the effective tensor is of interest, then the estimator was shown to be robust and can be used as a convergence criterion for the feedback procedure (note that there is no guaranty that this observation is true for other materials as it relies on the influence of the off-diagonal terms on the norm). In the following, we 
will consider the most unfavourable case as we will assume that we are interested in the value of all the components of $\mathcal{C}$ individually.

- As the estimator is not reliable in this case, a simple stagnation criterion will be used in the subsequent numerical experiments, as it is known to be a robust indicator ( $p-p-1$ error indicator). The convergence of $\mathcal{C}$ will be monitored through the convergence of its individual components (i.e. $\mathcal{C}$ is considered as converged if the stagnation criterion is met for all of them). Two stagnation criteria are considered: absolute and relative stagnations. Absolute stagnation is met if the variation of $C_{i j}$ between the two last computations is small with respect to the max-norm of $\mathcal{C}: \frac{C_{i j}^{h, p}-C_{i j}^{h, p-1}}{\left\|\mathcal{C}^{h, p-1}\right\|_{\max }}<\varepsilon_{a}$. Relative stagnation is met if $\epsilon\left(C_{i j}^{h, p}, C_{i j}^{h, p-1}\right)=$ $\frac{C_{i j}^{h, p}-C_{i j}^{h, p-1}}{C_{i j}^{h, p-1}}<\varepsilon_{r}$.

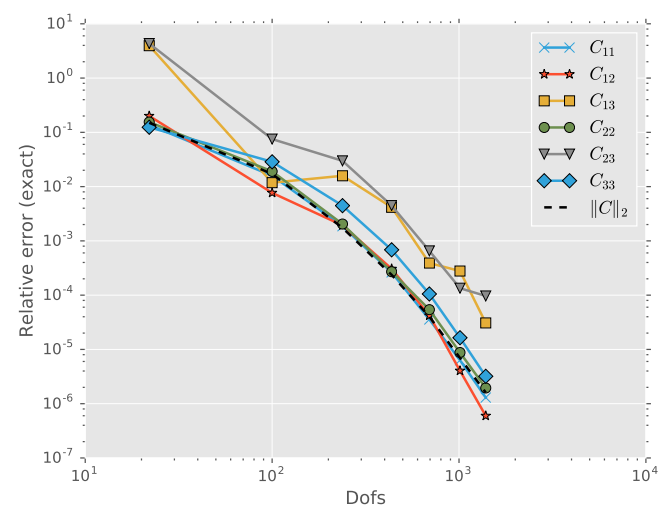

(a) Reference error

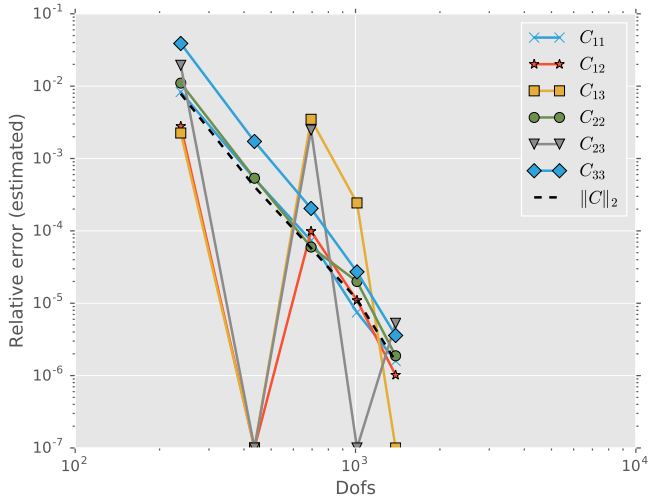

(b) Estimated error

Figure 5: 2D example: (a) reference errors $\epsilon\left(\mathcal{C}^{h, p}, \mathcal{C}^{\text {ref }}\right)$ and $\epsilon\left(\mathcal{C}_{i j}^{h, p}, \mathcal{C}_{i j}^{r e f}\right)$ and (b) estimated errors $\epsilon\left(\mathcal{C}^{h, p}, \mathcal{C}^{e s t}\right)$ and $\epsilon\left(\mathcal{C}_{i j}^{h, p}, \mathcal{C}_{i j}^{e s t}\right)$ of the homogenized tensor $\mathcal{C}$ and its components ( $p$ convergence).

\subsection{Deterministic Feedback}

Based on the conclusions of the last section, the adaptive determination of the homogenized tensor $\mathcal{C}$ is now considered. A non-conforming $p$ extension is used to make the overall properties converge. The convergence is monitored trough the stagnation of the components of $\mathcal{C}$. In this numerical example, the computational mesh contains $4 \times 4$ elements per side and the approximation can range from $p=1$ to $p=7$. The geometrical mesh is unchanged with respect to the previous section. Finally, the material properties are still considered as deterministic. The process is considered as converged if the relative stagnation of the components of $\mathcal{C}$ is lower than $\varepsilon_{r}=10^{-2}$ or $510^{-2}$, and 


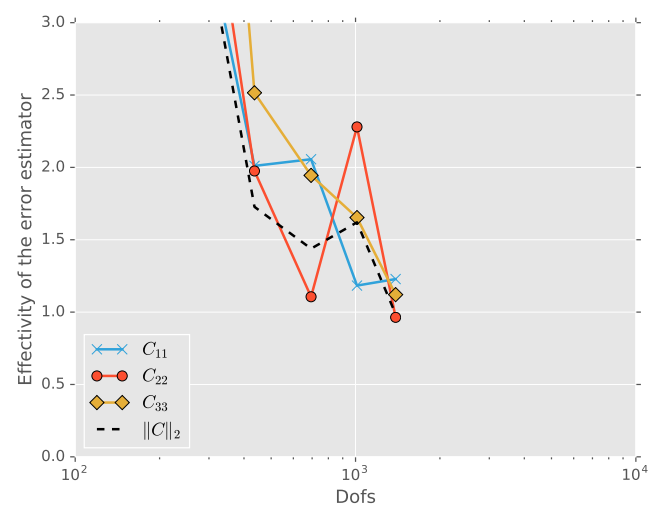

Figure 6: 2D example: Evolution of the effectivity of the estimators $\frac{\epsilon\left(\mathcal{C}_{i j}^{h, p}, \mathcal{C}_{i j}^{e s t}\right)}{\epsilon\left(\mathcal{C}_{i j}^{h, p}, \mathcal{C}_{i j}^{r e f}\right)}$ and $\frac{\epsilon\left(\mathcal{C}^{h, p}, \mathcal{C}^{e s t}\right)}{\epsilon\left(\mathcal{C}^{h, p}, \mathcal{C}^{r e f}\right)}(p$ convergence).

the absolute one lower than $\varepsilon_{a}=10^{-5}$. The evolution of the components of $\mathcal{C}$ during the procedure are depicted in figure 7 for these two different stagnation thresholds. It can be seen that the converged quantities can be obtained respectively for $p=5$ and $p=3$.

\subsection{Stochastic convergence}

The case of stochastic material properties is now considered. We first focus on the convergence of the stochastic homogenized quantities during $p$ refinement. The same porous problem is still considered, but now Young's modulus and Poisson's ratio are random. E ranges from $1 / 2$ to 1.5 , and $\nu$ from 0.15 to 0.45 both following a uniform probability distribution. Note that more complex probability distributions could be considered without any loss of generality, except unbounded distributions (like the Gaussian distribution). Indeed, negative values (for $E$ and $\nu$ ) and infinite (for $E$ ) or greater than $1 / 2$ values (for $\nu$ ) can occur with these probability distributions. A reference solution $\mathcal{C}^{\text {ref }}$ is obtained by considering an overkill solution, both in the deterministic and stochastic spaces. The deterministic overkill discretization is obtained by selecting 256 elements per side with quadratic elements and the stochastic one by means of two degree 10 polynomial chaos (Legendre polynomials) ${ }^{3}$ for $\mathcal{S}^{P_{1}}$ and $\mathcal{S}^{P_{2}}$. The final reference solution was obtained using 40 PGD modes. The $p$ convergence was conducted on a $4 \times 4$ elements mesh with $p$ from 1 to 7, and two quartic Legendre polynomial chaos for $E$ and $\nu$. At each step of the procedure, the reduced model approximates a spectral stochastic solution which should converge exponentially provided that the solution is smooth [75] in both space and stochastic spaces. This is consistent with the convergence results for the expectation and standard deviation of $\mathcal{C}$ shown in figure 8. All the components of $\operatorname{Exp}(\mathcal{C})$ and

\footnotetext{
${ }^{3}$ Note that high order approximations are used both in the deterministic and stochastic space.
} 


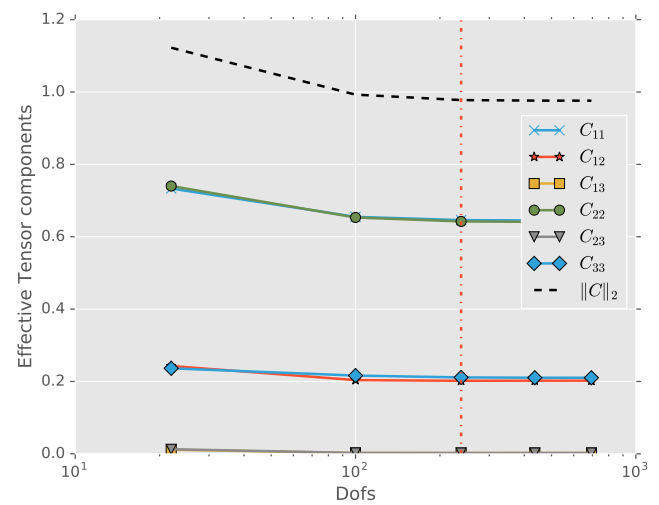

Figure 7: 2D example: Feedback history of the homogenized tensor $\mathcal{C}$. The thin vertical line indicates the fulfilment of the convergence criterion in the case of a $510^{-2}$ relative stagnation criterion.

$\operatorname{Std}(\mathcal{C})$ converge exponentially, which is also the case for the 2-norm of these tensors. As shown in the last section, the extrapolation error estimate cannot be used to drive the adaptation process if the accuracy of the individual components is mandatory. However, figure 9 shows that the robust deterministic behaviour of the estimator for the two-norm of $\mathcal{C}$ extends to the stochastic framework. It is important to note here that although $\operatorname{Exp}(E)$ and $\operatorname{Exp}(\nu)$ are equal to their deterministic counterparts, the expectation of the stochastic effective tensor is not equal to the deterministic one when mean values are used for both $E$ and $\nu$. This stems from the fact that the solution is nonlinear in $\nu$.

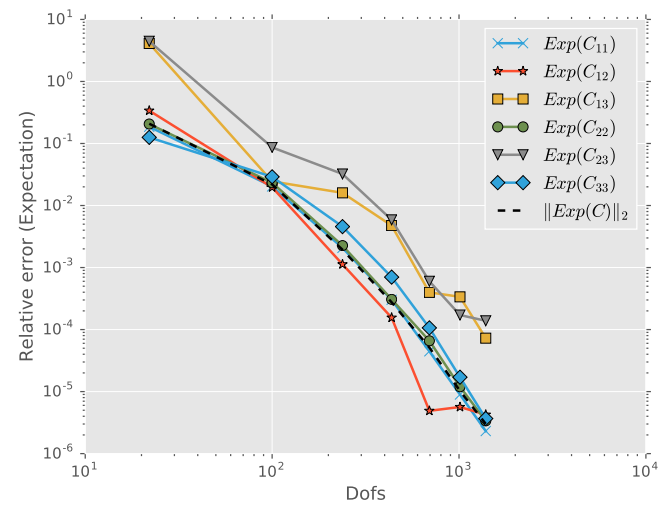

$\operatorname{Exp}(\mathcal{C})$

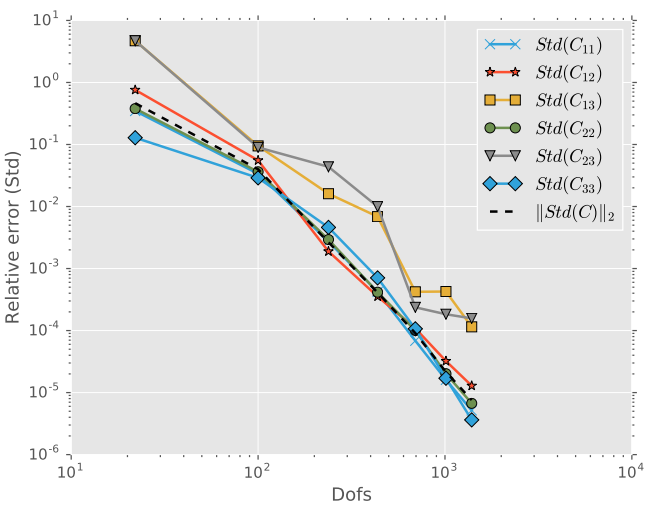

$\operatorname{Std}(\mathcal{C})$

Figure 8: 2D example: Evolution of $\epsilon\left(\operatorname{Exp}\left(\mathcal{C}_{i j}^{h, p}\right), \operatorname{Exp}\left(\mathcal{C}_{i j}^{r e f}\right)\right)$ and $\epsilon\left(\operatorname{Std}\left(\mathcal{C}_{i j}^{h, p}\right), \operatorname{Std}\left(\mathcal{C}_{i j}^{r e f}\right)\right)$ 


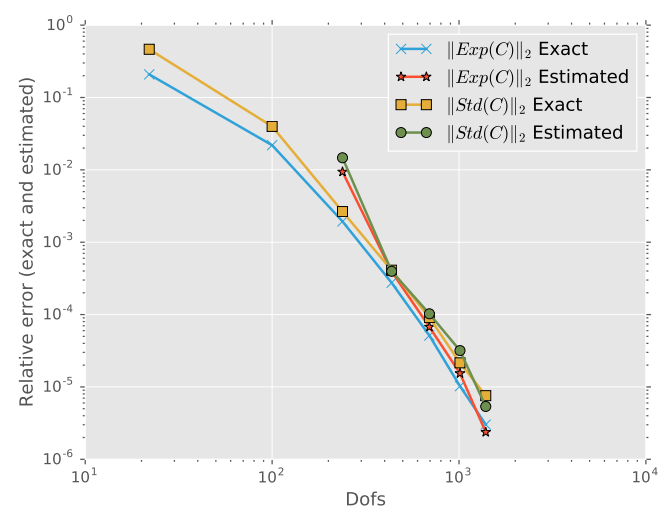

(a)

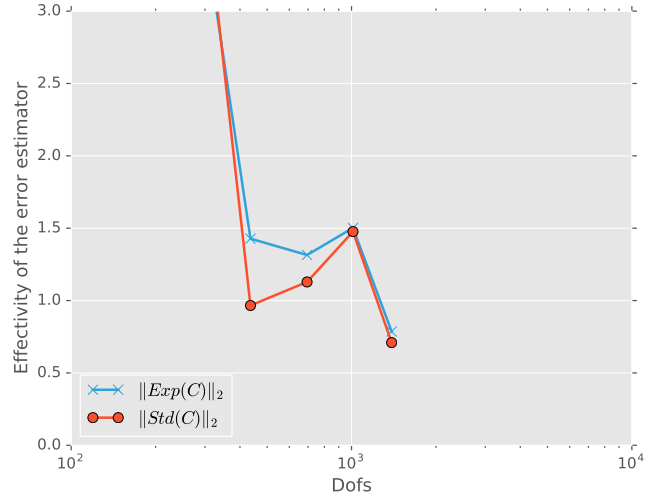

(b)

Figure 9: 2D example: (a) comparison between reference and estimated errors on $\operatorname{Exp}(\mathcal{C})$ and $\operatorname{Std}(\mathcal{C})\left(\epsilon\left(\operatorname{Exp}\left(\mathcal{C}^{h, p}\right), \operatorname{Exp}\left(\mathcal{C}^{r e f}\right)\right)\right.$ and $\left.\epsilon\left(\operatorname{Std}\left(\mathcal{C}^{h, p}\right), \operatorname{Std}\left(\mathcal{C}^{r e f}\right)\right)\right) ;(\mathrm{b})$ effectivity index.

\subsection{Stochastic Feedback}

Based on the verification of the convergence of the method, it is now possible to evaluate adaptively the expectation and standard deviation of $\mathcal{C}$. In this section, a $\varepsilon_{r}=10^{-2}$ threshold is considered for the relative stagnation, and $\varepsilon_{a}=10^{-5}$ for the absolute stagnation of both $\operatorname{Exp}(\mathcal{C})$ and $\operatorname{Std}(\mathcal{C})$. The evolution of the 2-norm of the expectation and standard deviation of the $\mathcal{C}$ are represented in figure 10. It can be seen that a $p=4$ spatial discretization and 13 PGD modes are sufficient to fall into this range of accuracy. Individual components are not presented here, but their evolution is similar to the one of the norm, even for the off-diagonal components. Next, the accuracy of the stochastic model is assessed. Indeed, this model allows to obtain the homogenized properties of the porous material for any value of $E$ and $\nu$ in their range of evolution. Thus, 10000 random evaluations of $E$ and $\nu$ are considered. From these random evaluations, $\mathcal{C}$ can be obtained either by using the reduced order model $\left(\mathcal{C}^{P G D}\right.$ with $m=13$ modes $)$ or by a direct computation of the overall properties $\left(\mathcal{C}^{M C}\right.$ for the same $h$ and $\left.p\right)$. The former approach is almost costless once the reduced order model has been computed while the second (Monte Carlo) is computationally demanding. The relative 2-norm error between these two tensors $\epsilon\left(\mathcal{C}^{P G D}, \mathcal{C}^{M C}\right)$ is evaluated for each sample and studied. The distribution of the error levels is then presented in figure 11. It can be seen that the error is smaller than $610^{-3}$, which is very accurate. Note also that the majority of the error levels are lower than $210^{-3}$. Finally, the reduced order model can be used to build the empirical probability density functions (pdf) of the components of $\mathcal{C}$. The pdf of selected components of $\mathcal{C}$ are compared to those obtained from 10000 direct Monte-Carlo simulations in figure 12. It can be seen that there is a perfect accordance between these 
curves $^{4}$, which highlights the accuracy if the proposed approach. Now that the method has been validated for simple problems, we consider its application to biomechanics.

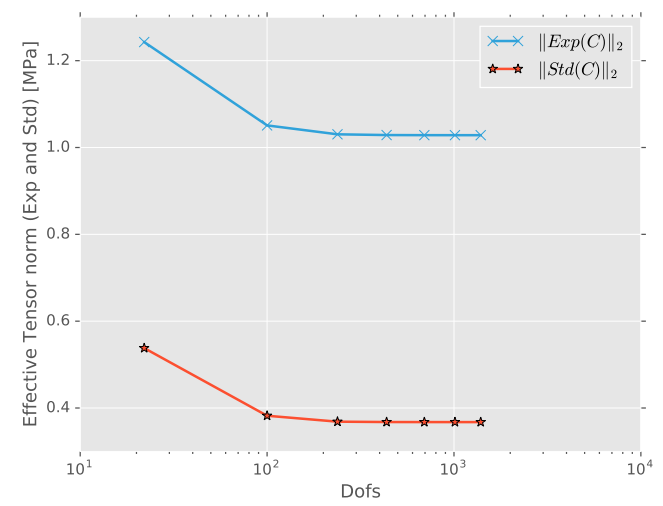

Figure 10: 2D example: Evolution of $\operatorname{Exp}(\mathcal{C})$ and $\operatorname{Std}(\mathcal{C})$ during the feedback procedure.

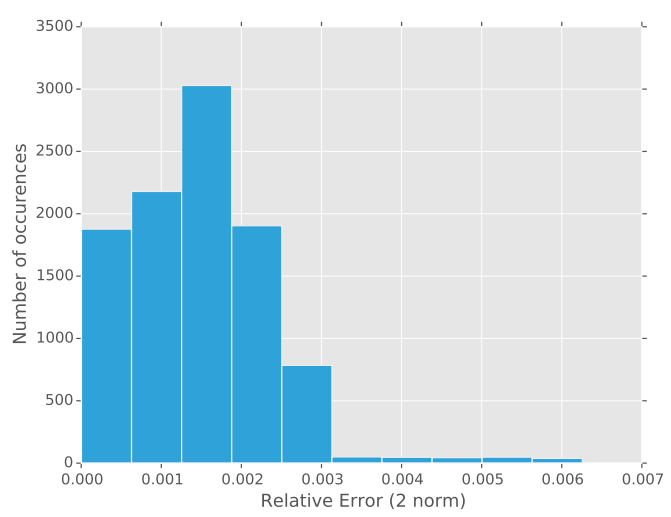

Figure 11: 2D example: Distribution of the error $\epsilon\left(\mathcal{C}^{P G D}, \mathcal{C}^{M C}\right)$ for 10000 random evaluations.

\section{Application to a real 3D bone micro-structure}

The method is now applied to a real bone micro-structure (see figure 13) whose volumic fraction is 0.1382 . The geometry was obtained by segmenting a bone CT scan following the procedure proposed in [40]. This raw CT scan is available on the Internet through the BEL Repository managed by the Istituti Ortopedici Rizzoli, Bologna, Italy. The

\footnotetext{
${ }^{4}$ It is also the case for the 3 other components that are not presented here.
} 

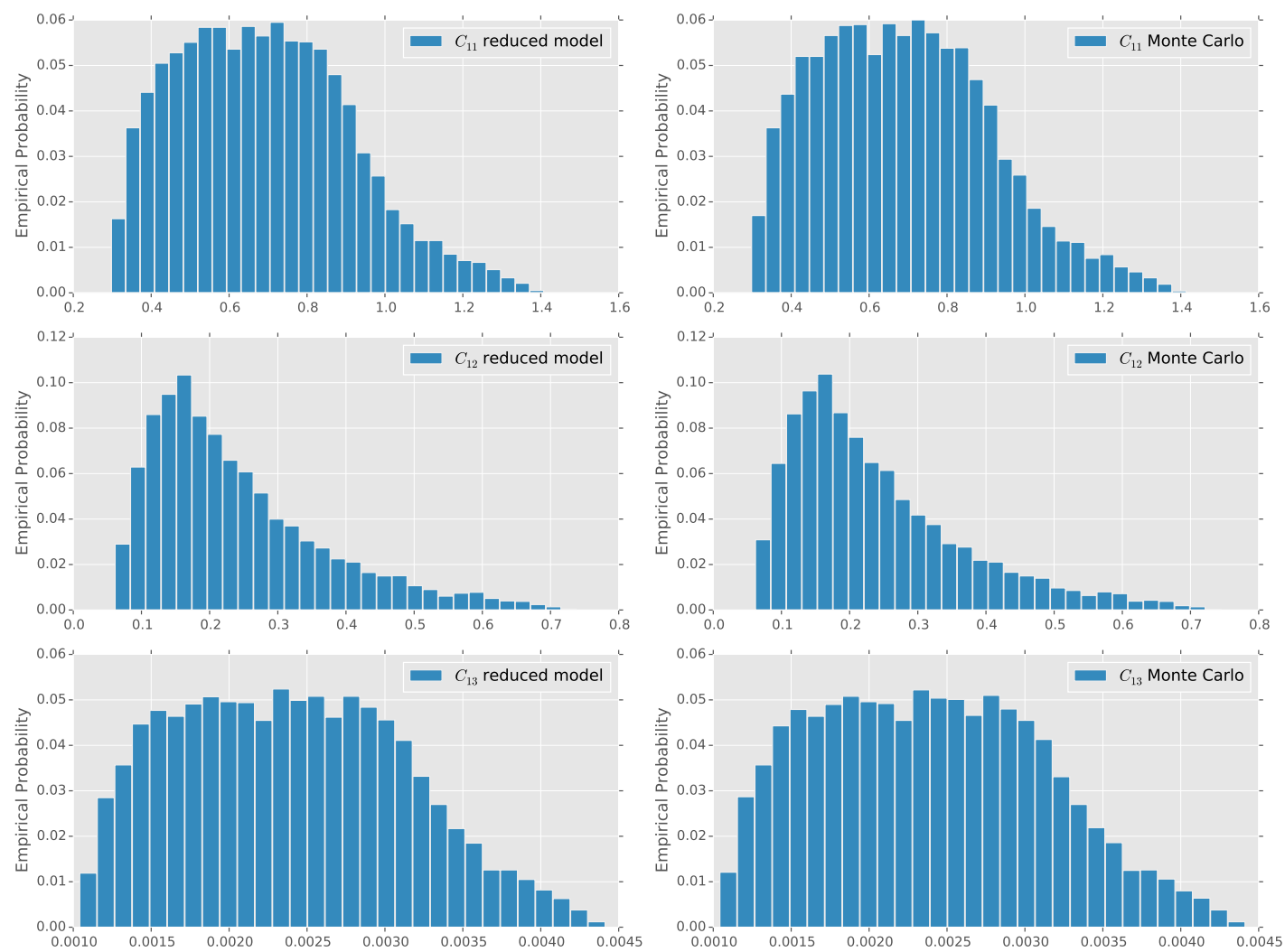

Figure 12: 2D example: Empirical pdf of the components of $\mathcal{C}$ : left, reduced model; right, Monte Carlo simulations (x-axis in $\mathrm{MPa}$ )

objective is to apply the proposed approach in order to compute the effective properties of this micro-structure. The material is considered as linear-elastic with typical material properties: $10000 \mathrm{MPa}$ Young's modulus and 0.4 Poisson's ratio. The resolution of the volume is $129^{3}$ voxels, which corresponds to a level 7 octree. Kinematic uniform boundary conditions are still used here in order to extract the homogenized behaviour of the bone. Note that this choice is known to lead to over-estimated values of the effective properties [47].

\subsection{Deterministic feedback}

The feedback method is first considered in order to evaluate the deterministic homogenized tensor $\mathcal{C}$. The computational mesh is made from a regular grid of 16 elements along each side of the cube, and the spatial approximation order ranges from $p=1$ to $p=8$. The following criteria are considered for stopping the refinement process: $\varepsilon_{r}=5 \%$ for relative stagnation and $\varepsilon_{a}=510^{-4}$ for absolute stagnation. It can be seen in figure 16 that these conditions are met for $p=5$, and that the method is able to evaluate efficiently 

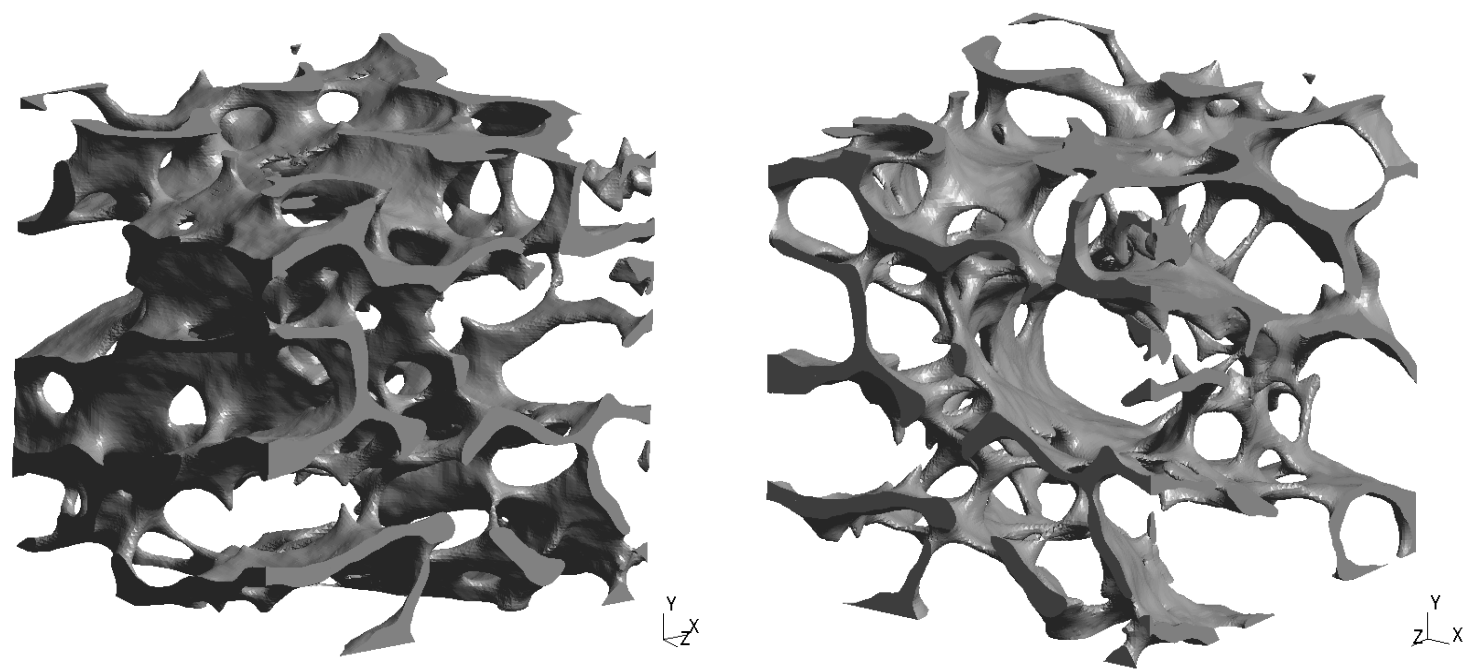

Figure 13: Bone geometry.

the effective properties of the material. Some corresponding micro-modes are presented in figures 14 and 15 for illustration. The results are consistent with the literature, as we found $C_{33}=727 \mathrm{Mpa}$, which is in agreement with the experimental results given in [2] (scattering between 200 and $900 \mathrm{Mpa}$ for this bone density).

\subsection{Stochastic feedback}

Finally, the evaluation of the stochastic effective properties of the bone is presented. Similarly to the $2 \mathrm{D}$ numerical examples, uniform probability distributions are considered. A large scattering is chosen for the material properties in order to highlight the accuracy of the method. Young's modulus thus ranges from $5000 \mathrm{MPa}$ to $15000 \mathrm{Mpa}$, and Poisson's ratio from 0.32 to 0.48 . Note that such a high Poisson's ratio would lead to some volumetric locking with low-order finite elements, which is not the case with high-order finite elements (see [31]). The convergence is attained when relative and absolute stagnation respectively falls below $\varepsilon_{r}=5 \%$ and $\varepsilon_{a}=510^{-4}$ for both expectation and standard deviation. This target accuracy is attained for $p=6$ and $m=13$ PGD modes. The evolution of the 2-norm of $\mathcal{C}$ and some of its components during the $p$ refinement process are depicted in figure 18. It can be observed that the largest dispersion is obtained for the diagonal part of the effective tensor. The expectation of $\mathcal{C}_{33}(729$ $\mathrm{Mpa}$ ) is very similar to the one obtained in the deterministic case. The corresponding standard deviation (211 Mpa) is smaller than the one highlighted in [2], as the range of variation of the parameters was not fitted with any experimental data. Note also that any comparison with [2] is difficult since the results are based on multiple donors whose age can have a large influence [76], and also because this study also includes experimental bias [77] that are out of the scope of this study. 


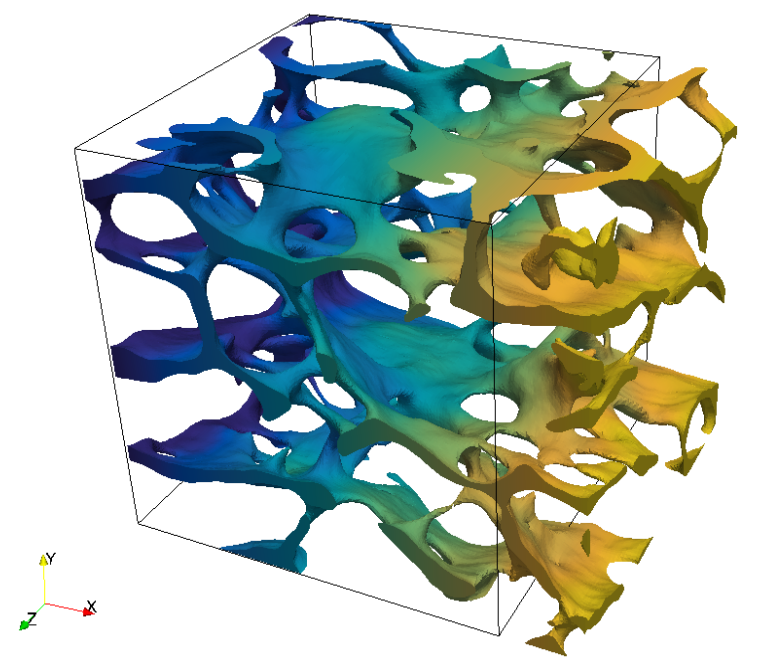

Mode 1

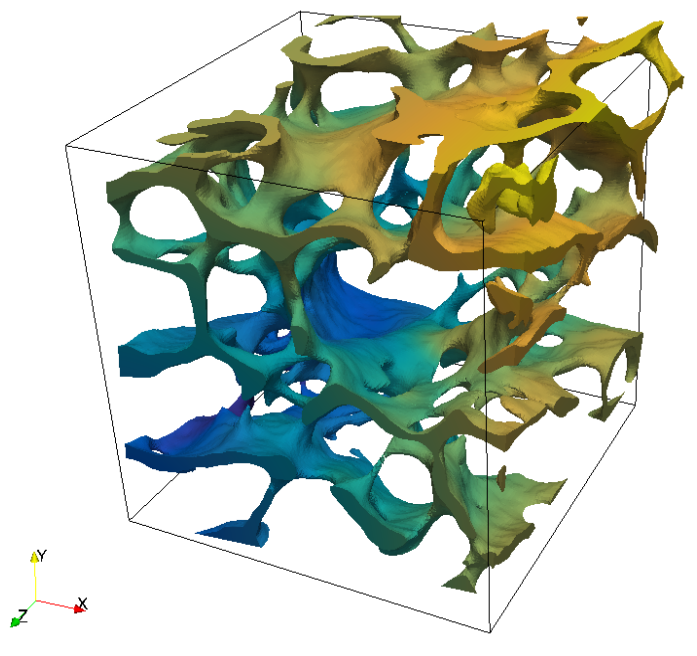

Mode 4

Figure 14: Micro-modes 1 (left) and 4 (right): displacement field

The accuracy of the reduced model is now assessed through 100 samplings of the material properties. The reduced order model is particularized for these 100 samplings, and the results are compared with the corresponding direct calculations. The error distribution $\epsilon\left(\mathcal{C}^{P G D}, \mathcal{C}^{M C}\right)$ is plotted in figure 19: it can be seen that it is smaller than $1.510^{-3}$, which highlights the accuracy of the reduced model. For a finer comparison between direct and reduced solutions, we now consider the mechanical fields associated to a given sample. The local relative error on the von-mises stress between direct and reduced solutions is presented in figure 17 where it can be seen that this local error is very small.

Finally, the reduced model is used to build an empirical probability density function: 10000 samples are generated at no additional cost. The empirical pdf of the different components of the tensor are presented in figure 20. 


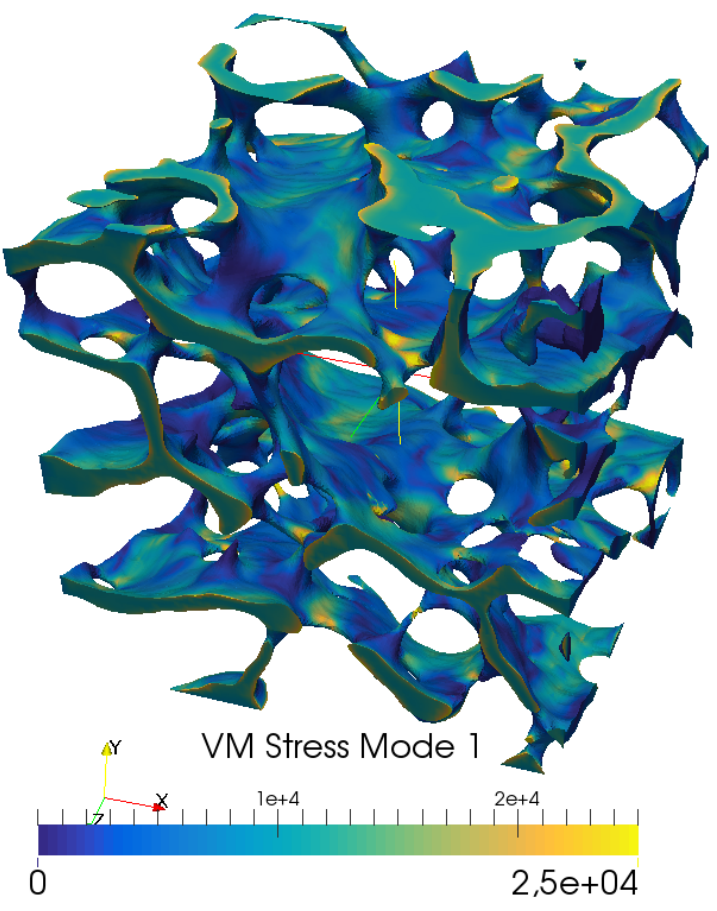

Mode 1

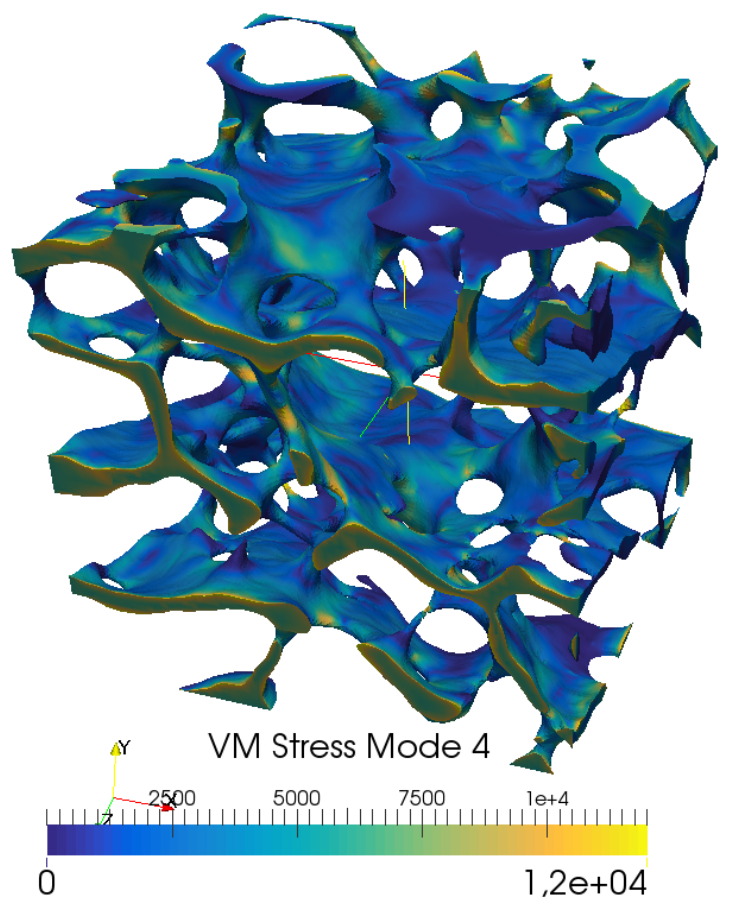

Mode 4

Figure 15: Micro-modes 1 (left) and 4 (right): Von-Mises stress field

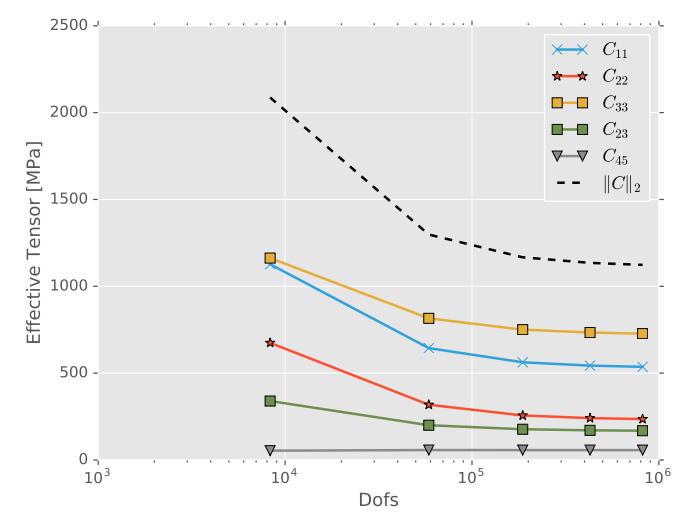

Figure 16: 3D example: Evolution of some components of $\mathcal{C}$ during the feedback procedure. 


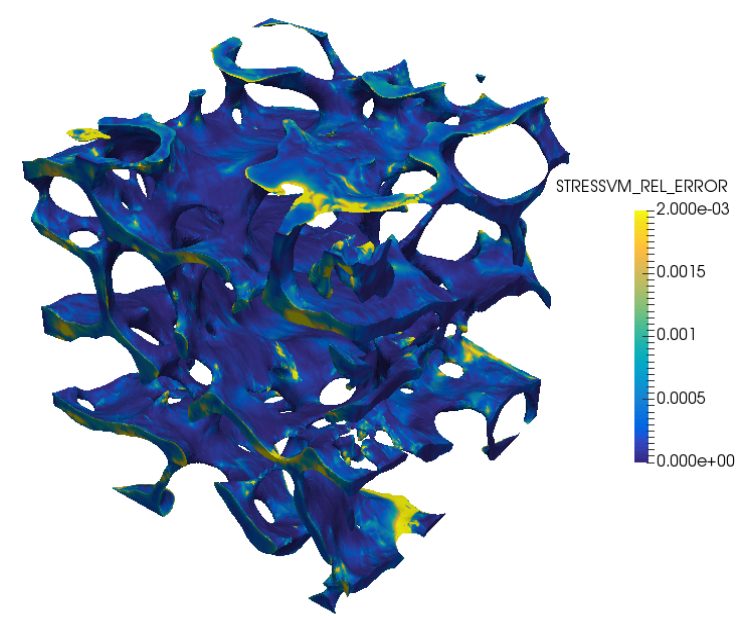

Figure 17: 3D example: Local relative error between direct and reduced solution for a given sample.

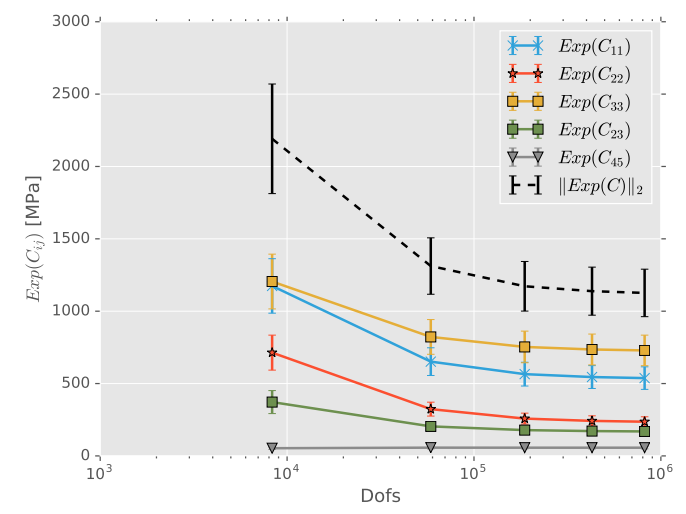

Figure 18: 3D example: Evolution of some of the components of $\operatorname{Exp}(\mathcal{C})$ and associated dispersion during the feedback procedure. 


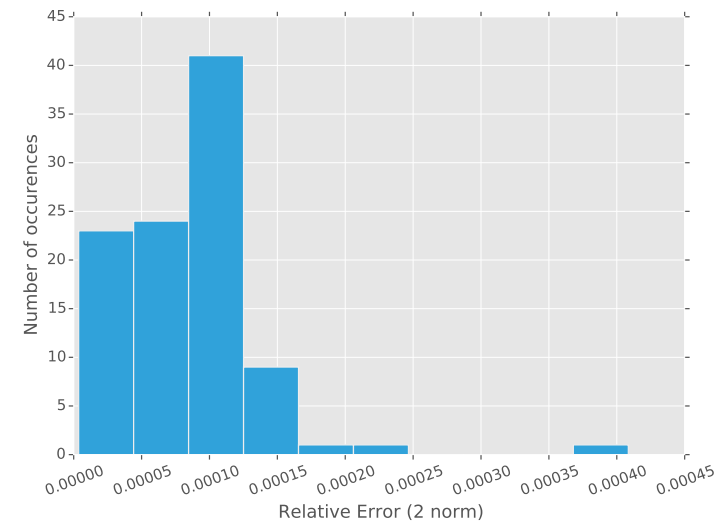

Figure 19: 3D example: Distribution of the error $\epsilon\left(\mathcal{C}^{P G D}, \mathcal{C}^{M C}\right)$ for 100 random evaluations.

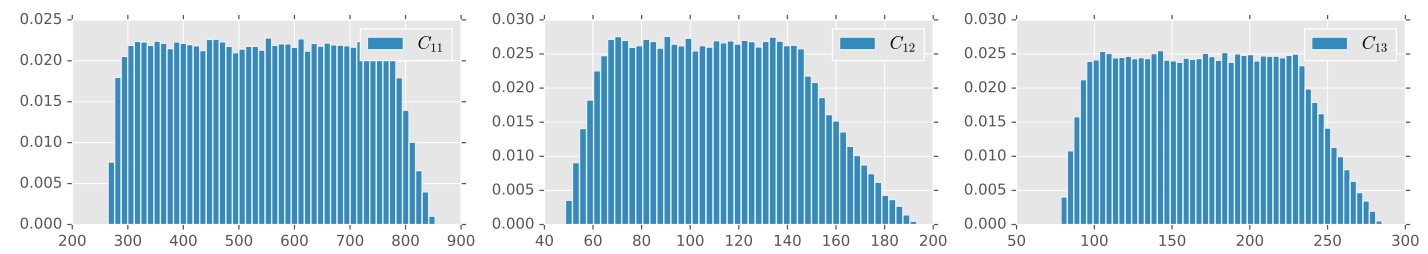

Figure 20: 3D example: Empirical pdf of the components of $\mathcal{C}$ (x-axis in $\mathrm{MPa}$ ). 


\section{Conclusion}

An innovative numerical strategy has been proposed in order to compute adaptively the effective properties of complex micro-structures with uncertain material properties. The method relies on two main ingredients: (i) a high-order fictitious domain method approximation which enables to avoid the meshing burden with such complex geometries, and (ii) a model reduction technique based on the Proper Generalized Decomposition of the spectral stochastic representation of uncertainties (Linear Elasticity was considered here, with random Young's modulus and Poisson's ratio). The accuracy of the output was ensured even with large uncertainties on the material properties thanks to a feedback method which is used to monitor the convergence of the effective properties. The method has been verified on a simple 2D example which has shown that error estimates based on extensions were not sufficiently reliable for this type of application. However, it was seen that the use of a stagnation criterion allowed to obtain very accurate results as both expectation and standard deviation errors converge exponentially upon $p$ refinement. The resulting stochastic effective tensor was seen to match Monte-Carlo's results with only a fraction of the computational cost. The method was finally applied to a real bone micro-structure whose stochastic effective properties could be obtained very efficiently. In order to improve the proposed strategy, goal oriented error estimation could be considered, as in $[52,38]$ for the deterministic side, but also on the stochastic side. Note however that the influence of the stochastic discretization on the computational cost is very low thanks to the model reduction approach. Finally, multi-material and/or nonlinear materials could be considered, as well as the influence of a possible geometrical randomness.

Acknowledgements: G.Legrain and N. Takano would like to acknowledge partial support of the Erasmus Mundus BEAM program.

\section{A. Derivation of the reduced model}

Recall the minimization problem (29). Two independent random variables are considered: $\xi_{1}$ for Young's modulus and $\xi_{2}$ for Poisson's ratio. Using a progressive definition of the PGD, we look for a solution in the form: $\mathbf{u}_{m}=\mathbf{u}_{m-1}+\mathbf{w}(\mathbf{x}) \phi^{1}\left(\xi_{1}\right) \phi^{2}\left(\xi_{2}\right)$ where an element $\mathbf{w}(\mathbf{x}) \phi^{1}\left(\xi_{1}\right) \phi^{2}\left(\xi_{2}\right)$ is solution of problem (31). Functional $J\left(\mathbf{u}_{m-1}+\mathbf{w} \phi^{1} \phi^{2}\right)$ 
can be expanded into:

$$
\begin{aligned}
J\left(\mathbf{u}_{m-1}+\mathbf{w} \phi^{1} \phi^{2}\right) & =\frac{1}{2} A\left(\mathbf{u}_{m-1}+\mathbf{w} \phi^{1} \phi^{2}, \mathbf{u}_{m-1}+\mathbf{w} \phi^{1} \phi^{2}\right)-B\left(\mathbf{w} \phi^{1} \phi^{2}\right) \\
& =\frac{1}{2} \int_{\theta_{1}} \int_{\theta_{2}} \int_{\Omega} \underline{\underline{\varepsilon}}\left(\mathbf{w} \phi^{1} \phi^{2}\right) \mathcal{K}\left(\mathbf{x}, \xi_{1}, \xi_{2}\right) \underline{\underline{\varepsilon}}\left(\mathbf{w} \phi^{1} \phi^{2}\right) d \Omega d P_{\xi_{2}} d P_{\xi_{1}} \\
& -\left(\int_{\theta_{1}} \int_{\theta_{2}} \int_{\Omega} \mathbf{b}(\mathbf{x}) \mathbf{w}(\mathbf{x}) \phi^{1} \phi^{2} d \Omega d P_{\xi_{2}} d P_{\xi_{1}}\right. \\
& \left.-\frac{1}{2} A\left(\mathbf{u}_{m-1}, \mathbf{u}_{m-1}\right)-A\left(\mathbf{u}_{m-1}, \mathbf{w} \phi^{1} \phi^{2}\right)\right)
\end{aligned}
$$

where the last terms in parenthesis are denoted as $R\left(\mathbf{u}_{m-1}, \mathbf{b}(\mathbf{x}), \mathbf{w}(\mathbf{x}) \phi^{1} \phi^{2}\right)$.

In addition, we can write:

$$
\underline{\underline{\varepsilon}}\left(\mathbf{w}(\mathbf{x}) \phi^{1}\left(\xi_{1}\right) \phi^{2}\left(\xi_{2}\right)\right)=\phi^{1}\left(\xi_{1}\right) \phi^{2}\left(\xi_{2}\right) \underline{\underline{\varepsilon}}(\mathbf{w}(\mathbf{x})) .
$$

In our case, the Hooke tensor can be separated as:

$$
\mathcal{K}\left(\mathbf{x}, \xi_{1}, \xi_{2}\right)=\lambda\left(\xi_{1}, \xi_{2}\right) \mathcal{K}_{\lambda}(\mathbf{x})+\mu\left(\xi_{1}, \xi_{2}\right) \mathcal{K}_{\mu}(\mathbf{x})
$$

with:

$$
\begin{aligned}
\lambda\left(\xi_{1}, \xi_{2}\right) & =\frac{\xi_{1} \xi_{2}}{\left(1+\xi_{2}\right)\left(1-2 \xi_{2}\right)}, \\
\mu\left(\xi_{1}, \xi_{2}\right) & =\frac{\xi_{1}}{2\left(1+\xi_{2}\right)} .
\end{aligned}
$$

where $\xi_{1}=E$ and $\xi_{2}=\nu$. Thus, we have:

$$
\begin{aligned}
\mathcal{K}\left(\mathbf{x}, \xi_{1}, \xi_{2}\right) & =\xi_{1} \frac{\xi_{2}}{\left(1+\xi_{2}\right)\left(1-2 \xi_{2}\right)} \mathcal{K}_{\lambda}(\mathbf{x})+\xi_{1} \frac{1}{2\left(1+\xi_{2}\right)} \mathcal{K}_{\mu}(\mathbf{x}) \\
& =\mathcal{K}_{1}^{1}\left(\xi_{1}\right) \mathcal{K}_{1}^{2}\left(\xi_{2}\right) \mathcal{K}_{1}^{x}(\mathbf{x})+\mathcal{K}_{2}^{1}\left(\xi_{1}\right) \mathcal{K}_{2}^{2}\left(\xi_{2}\right) \mathcal{K}_{2}^{x}(\mathbf{x})
\end{aligned}
$$

All the integrals can be separated, leading to the following functional:

$$
\begin{array}{r}
J\left(\mathbf{u}_{m-1}+\mathbf{w} \phi^{1} \phi^{2}\right)= \\
\frac{1}{2} \int_{\theta_{1}} \phi^{1}\left(\xi_{1}\right) \mathcal{K}_{1}^{1}\left(\xi_{1}\right) \phi^{1}\left(\xi_{1}\right) d P_{\xi_{1}} \int_{\theta_{2}} \phi^{1}\left(\xi_{1}\right) \mathcal{K}_{1}^{2}\left(\xi_{2}\right) \phi^{2}\left(\xi_{2}\right) d P_{\xi_{2}} \int_{\Omega} \underline{\underline{\varepsilon}}(\mathbf{w}(\mathbf{x})) \mathcal{K}_{1}^{x}(\mathbf{x}) \underline{\underline{\varepsilon}}(\mathbf{w}(\mathbf{x})) d \Omega+ \\
\frac{1}{2} \int_{\theta_{1}} \phi^{1}\left(\xi_{1}\right) \mathcal{K}_{2}^{1}\left(\xi_{1}\right) \phi^{1}\left(\xi_{1}\right) d P_{\xi_{1}} \int_{\theta_{2}} \phi^{1}\left(\xi_{1}\right) \mathcal{K}_{2}^{2}\left(\xi_{2}\right) \phi^{2}\left(\xi_{2}\right) d P_{\xi_{2}} \int_{\Omega} \underline{\underline{\varepsilon}}(\mathbf{w}(\mathbf{x})) \mathcal{K}_{2}^{x}(\mathbf{x}) \underline{\underline{\varepsilon}}(\mathbf{w}(\mathbf{x})) d \Omega \\
-R\left(\mathbf{u}_{m-1}, \mathbf{b}(\mathbf{x}), \mathbf{w}(\mathbf{x}) \phi^{1}\left(\xi_{1}\right) \phi^{2}\left(\xi_{2}\right)\right)
\end{array}
$$

The expression of $R$ is not given here, but can be obtained following the same rationale. Necessary conditions of optimality for a triplet $\left(\mathbf{w}, \phi^{1}, \phi^{2}\right)$ are the three Galerkin criteria:

$$
\begin{aligned}
& \phi^{1}=\arg \min _{\chi^{1} \in \mathcal{S}^{N_{1}}} J\left(\mathbf{u}_{m-1}+\mathbf{w} \chi^{1} \phi^{2}\right) \\
& \phi^{2}=\arg \min _{\chi^{2} \in \mathcal{S}^{N_{2}}} J\left(\mathbf{u}_{m-1}+\mathbf{w} \phi^{1} \chi^{2}\right) \\
& \mathbf{w}=\arg \min _{\mathbf{Y} \in \mathcal{V}^{N}} J\left(\mathbf{u}_{m-1}+\mathbf{Y} \phi^{1} \phi^{2}\right)
\end{aligned}
$$


Thus, the nonlinear equation is solved by means of a fixed point algorithm which reduces in the following three linear problems:

$P_{1}: \phi^{2}$ and $\mathbf{w}$ are known. $\phi^{1}$ is solution of problem (41). Stochastic problem of dimension $\operatorname{dim}\left(\mathcal{S}^{N_{1}}\right)$

$P_{2}: \phi^{1}$ and $\mathbf{w}$ are known. $\phi^{2}$ is solution of problem (42). Stochastic problem of dimension $\operatorname{dim}\left(\mathcal{S}^{N_{2}}\right)$;

$P_{3}: \phi^{1}$ and $\phi^{2}$ are known. $\mathbf{w}$ is solution of problem (43). Stochastic problem of dimension $\operatorname{dim}\left(\mathcal{V}^{N}\right)$

Additional update strategies can be considered in order to improve the efficiency of the algorithm, the interested reader can refer to [73, 67, 55].

\section{References}

[1] J.H. Keyak, J.M Meagher, H.B. Skinner, and C.D Mote Jr. Automated threedimensional finite element modelling of bone: a new method. Journal of Biomedical Engineering, 12(5):389-397, 1990.

[2] J.H. Keyak, I.Y. Lee, and H.B. Skinner. Correlations between orthogonal mechanical properties and density of trabecular bone: Use of different densitometric measures. Journal of biomedical materials research, 28:1329-1336, 1994.

[3] Domenico Bongini, Monica Carfagni, and Lapo Governi. Hippin: A semiautomatic computer program for selecting hip prosthesis femoral components. Computer Methods and Programs in Biomedicine, 63(2):105-115, 2000.

[4] Christian Dick, Joachim Georgii, Rainer Burgkart, and Rüdiger Westermann. Stress tensor field visualization for implant planning in orthopedics. IEEE Transactions on Visualization and Computer Graphics, 15(6):1399-1406, 2009.

[5] Kenjiro Terada, T Miura, and Noboru Kikuchi. Digital image-based model- ing applied to the homogenization analysis of composite materials. Computational Mechanics, 20:331-346, 1997.

[6] Valerie R. Coffman, Andrew C E Reid, Stephen a. Langer, and Gunay Dogan. OOF3D: An image-based finite element solver for materials science. Mathematics and Computers in Simulation, 82(12):2951-2961, 2012.

[7] B van Rietbergen, H Weinans, R Huiskes, and A Odgaard. A new method to determine trabecular bone elastic properties and loading using micromechanical finite-element models. Journal of biomechanics, 28(1):69-81, 1995.

[8] S.J Hollister and Noboru Kikuchi. Homogenization theory and digital imaging: a basis for studying the mechanics and design principles of bone tissue. Biotechnology and Bioengineering, 43(7):586-596, 1994. 
[9] Naoki Takano, M Zako, F Kubo, and K Kimura. Microstructure-based stress analysis and evaluation for porous ceramics by homogenization method with digital image-based modeling. International Journal of Solids and Structures, 40(5):1225$1242,2003$.

[10] Khairul Salleh Basaruddin, Naoki Takano, and Takayoshi Nakano. Stochastic multiscale prediction on the apparent elastic moduli of trabecular bone considering uncertainties of biological apatite (BAp) crystallite orientation and image-based modelling. Computer methods in biomechanics and biomedical engineering, 18(2):162174, apr 2015.

[11] W. D. Lian, Grégory Legrain, and Patrice Cartraud. Image-based computational homogenization and localization: comparison between X-FEM/levelset and voxelbased approaches. Computational Mechanics, 51(3):279-293, may 2013.

[12] Pascal Frey, B Sarter, and M Gautherie. Fully automatic mesh generation for 3-D domains based upon voxel sets. International Journal for Numerical Methods in Engineering, 37(16):2735-2754, 1994.

[13] D Ulrich, B van Rietbergen, H Weinans, P Rüegsegger, and P Regsegger. Finite element analysis of trabecular bone structure: a comparison of image-based meshing techniques. Journal of biomechanics, 31(12):1187-92, dec 1998.

[14] E Maire, A Fazekas, L Salvo, S Youssef, P Cloetens, and J.M Letang. X-ray tomography applied to the characterization of cellular materials related finite element modeling problems. Composites science and technology, 63(16):2431-2443, 2003.

[15] Alexander Düster, Jamshid Parvizian, Z Yang, and Ernst Rank. The finite cell method for three-dimensional problems of solid mechanics. Computer Methods in Applied Mechanics and Engineering, 197(45-48):3768-3782, 2008.

[16] Ernst Rank, Stefan Kollmannsberger, Ch. Sorger, and Alexander Düster. Shell Finite Cell Method: A high order fictitious domain approach for thin-walled structures. Computer Methods in Applied Mechanics and Engineering, 200(45-46):32003209 , oct 2011.

[17] Jamshid Parvizian, Alexander Düster, and Ernst Rank. Finite cell method - h and $\mathrm{p}$ extension for embedded domain problems in solid mechanics. Computational Mechanics, 41(1):121-133, 2007.

[18] Dominik Schillinger, Martin Ruess, Nils Zander, Yuri Bazilevs, Alexander Düster, and Ernst Rank. Small and large deformation analysis with the p- and B-spline versions of the Finite Cell Method. Computational Mechanics, 50(4):445-478, feb 2012.

[19] Nicolas Moës, John E. Dolbow, and Ted Belytschko. A finite element method for crack growth without remeshing. International Journal for Numerical Methods in Engineering, 46:131-150, 1999. 
[20] N. Sukumar, Nicolas Moës, Ted Belytschko, and B. Moran. Extended Finite Element Method for three-dimensional crack modelling. International Journal for Numerical Methods in Engineering, 48(11):1549-1570, 2000.

[21] M Stolarska, D L Chopp, Nicolas Moës, and T Belytschko. Modelling Crack Growth by Level Sets and the Extended Finite Element Method. International Journal for Numerical Methods in Engineering, 51(8):943-960, 2001.

[22] N. Sukumar, D. L. Chopp, Nicolas Moës, and Ted Belytschko. Modeling Holes and Inclusions by Level Sets in the Extended Finite Element Method. Comp. Meth. in Applied Mech. and Engrg., 190:6183-6200, 2001.

[23] N. Sukumar, D. L. Chopp, Nicolas Moës, and Ted Belytschko. Modeling holes and inclusions by level sets in the extended finite-element method. Computer Methods in Applied Mechanics and Engineering, 190(46-47):6183-6200, 2001.

[24] Nicolas Moës, A. Gravouil, and Ted Belytschko. Non-planar 3D crack growth by the extended finite element and level sets-Part I: Mechanical model. International Journal for Numerical Methods in Engineering, 53(11):2549-2568, 2002.

[25] Anthony Gravouil, Nicolas Moës, and Ted Belytschko. Non-planar 3D crack growth by the extended finite element and level sets. Part II: level set update. International Journal for Numerical Methods in Engineering, 53(11):2569-2586, apr 2002.

[26] Nicolas Moës, Mathieu Cloirec, Patrice Cartraud, and Jean-François Remacle. A computational approach to handle complex microstructure geometries. Comp. Meth. in Applied Mech. and Engrg., 192:3163-3177, 2003.

[27] Grégory Legrain, Nicolas Moës, and E. Verron. Numerical simulation of large strain fracture problems using X-FEM. International Journal for Numerical Methods in Engineering, 63(2):290-314, may 2005.

[28] Nicolas Moës, Éric Béchet, and Matthieu Tourbier. Imposing Dirichlet boundary conditions in the extended finite element method. International Journal for $\mathrm{Nu}$ merical Methods in Engineering, 67(12):1641-1669, 2006.

[29] Grégory Legrain, Nicolas Moës, and Antonio Huerta. Stability of incompressible formulations enriched with X-FEM. Computer Methods in Applied Mechanics and Engineering, 197(21-24):1835-1849, 2008.

[30] Grégory Legrain and Nicolas Moës. Treatment of nearly-singular problems with the X-FEM. Advanced Modeling and Simulation in Engineering Sciences, 1(1):13, dec 2014.

[31] Ivo Babuška and Barna Szabó. On the rates of convergence of the finite element method. International Journal for Numerical Methods in Engineering, 18(November 1980):323-341, 1982. 
[32] Barna Szabó. The p- and h-p versions of the finite element method in solid mechanics. Comp. Meth. in Applied Mech. and Engrg., 80:185-195, 1990.

[33] Barna Szabó and Ivo Babuška. Finite Element Analysis. John Wiley \& Sons, 1 edition, 1991.

[34] Ernst Rank, M. Ruess, Stefan Kollmannsberger, Dominik Schillinger, and Alexander Düster. Geometric modeling, isogeometric analysis and the finite cell method. Computer Methods in Applied Mechanics and Engineering, 249-252:104-115, dec 2012.

[35] Alexander Düster, Hans-Georg Sehlhorst, and Ernst Rank. Numerical homogenization of heterogeneous and cellular materials utilizing the finite cell method. Computational Mechanics, 50(4):413-431, jan 2012.

[36] Stephan Heinze, Meysam Joulaian, and Alexander Düster. Numerical homogenization of hybrid metal foams using the finite cell method. Computers \& Mathematics with Applications, 2015.

[37] Martin Ruess, David Tal, Nir Trabelsi, Zohar Yosibash, and Ernst Rank. The finite cell method for bone simulations: Verification and validation. Biomechanics and Modeling in Mechanobiology, 11(3-4):425-437, 2012.

[38] Clemens V. Verhoosel, G.J. van Zwieten, B. van Rietbergen, and R. de Borst. Imagebased goal-oriented adaptive isogeometric analysis with application to the micromechanical modeling of trabecular bone. Computer Methods in Applied Mechanics and Engineering, jul 2014.

[39] Ted Belytschko, Chandu Parimi, Nicolas Moës, N. Sukumar, and Shuji Usui. Structured extended finite element methods for solids defined by implicit surfaces. International Journal for Numerical Methods in Engineering, 56(4):609-635, jan 2003.

[40] Grégory Legrain, Patrice Cartraud, I Perreard, and Nicolas Moës. An X-FEM and level set computational approach for image-based modelling: Application to homogenization. International Journal for Numerical Methods in Engineering, 86(7):915934, 2011.

[41] Grégory Legrain, R Allais, and Patrice Cartraud. On the use of the extended finite element method with quadtree/octree meshes. International Journal for Numerical Methods in Engineering, 86(6):717-743, may 2011.

[42] Kristell Dréau, Nicolas Chevaugeon, and Nicolas Moës. Studied X-FEM enrichment to handle material interfaces with higher order finite element. Computer Methods in Applied Mechanics and Engineering, 199(29-32):1922-1936, jun 2010.

[43] Grégory Legrain, Nicolas Chevaugeon, and Kristell Dréau. High order X-FEM and levelsets for complex microstructures: Uncoupling geometry and approximation. Computer Methods in Applied Mechanics and Engineering, 241-244:172-189, oct 2012 . 
[44] Badri Hiriyur, Haim Waisman, and George Deodatis. Uncertainty quantification in homogenization of heterogeneous microstructures modeled by XFEM. International Journal for Numerical Methods in Engineering, (March):257-278, 2011.

[45] Dimitris Savvas, George Stefanou, Manolis Papadrakakis, and George Deodatis. Homogenization of random heterogeneous media with inclusions of arbitrary shape modeled by XFEM. Computational Mechanics, 54(5):1221-1235, jul 2014.

[46] T. Kanit, S. Forest, I. Galliet, V. Mounoury, and D. Jeulin. Determination of the size of the representative volume element for random composites: statistical and numerical approach. International Journal of Solids and Structures, 40(1314):3647-3679, jun 2003.

[47] C. Huet. Application of variational concepts to size effects in elastic heterogeneous bodies. Journal of the Mechanics and Physics of Solids, 38(6):813-841, 1990.

[48] Martin Ostoja-Starzewski. Material spatial randomness: From statistical to representative volume element. Probabilistic Engineering Mechanics, 21(2):112-132, 2006.

[49] Hagen Wille, Ernst Rank, and Zohar Yosibash. Prediction of the mechanical response of the femur with uncertain elastic properties. Journal of biomechanics, 45(7):1140-8, apr 2012.

[50] Roger G. Ghanem and Pol D. Spanos. Stochastic Finite Elements: A Spectral Approach. Springer New York, New York, NY, 1991.

[51] H.G. Matthies. Stochastic finite elements: Computational approaches to stochastic partial differential equations. ZAMM, 88(11):849-873, nov 2008.

[52] José Miranda Guedes, Noboru Kikuchi, Applied Mechanics, and Ann Arbor. Preprocessing and postprocessing for materials based on the homogenization method with adaptive finite element methods. Computer Methods in Applied Mechanics and Engineering, 83:143-198, 1990.

[53] Amine Ammar, B. Mokdad, F. Chinesta, and R. Keunings. A new family of solvers for some classes of multidimensional partial differential equations encountered in kinetic theory modeling of complex fluids. Journal of Non-Newtonian Fluid Mechanics, 139(3):153-176, dec 2006.

[54] Francisco Chinesta, Amine Ammar, and Elías Cueto. Recent Advances and New Challenges in the Use of the Proper Generalized Decomposition for Solving Multidimensional Models. Archives of Computational Methods in Engineering, 17(4):327350 , oct 2010 .

[55] Anthony Nouy. A priori model reduction through Proper Generalized Decomposition for solving time-dependent partial differential equations. Computer Methods in Applied Mechanics and Engineering, 199(23-24):1603-1626, 2010. 
[56] Dominik Schillinger and Ernst Rank. An unfitted hp-adaptive finite element method based on hierarchical B-splines for interface problems of complex geometry. Computer Methods in Applied Mechanics and Engineering, 200(47-48):3358-3380, 2011.

[57] J A Sethian. Level Set Methods and Fast Marching Methods: Evolving Interfaces in Computational Geometry; Fluid Mechanics; Computer Vision; and Material Science. Cambridge University Press, 1996.

[58] Kwok Wah Cheng and Thomas-Peter Fries. Higher-order XFEM for curved strong and weak discontinuities. International Journal for Numerical Methods in Engineering, pages $\mathrm{n} / \mathrm{a}-\mathrm{n} / \mathrm{a}, 2009$.

[59] Grégory Legrain. A NURBS enhanced extended finite element approach for unfitted CAD analysis. Computational Mechanics, 52(4):913-929, apr 2013.

[60] László Kudela, Nils Zander, Tino Bog, Stefan Kollmannsberger, and Ernst Rank. Efficient and accurate numerical quadrature for immersed boundary methods. Advanced Modeling and Simulation in Engineering Sciences, 2(1):10, 2015.

[61] Thomas-Peter Fries and Samir Omerović. Higher-order accurate integration of implicit geometries. International Journal for Numerical Methods in Engineering, pages $n / a-n / a, 2015$.

[62] Onofre Marco, Ruben Sevilla, Yongjie Zhang, Juan José Ródenas, and Manuel Tur. Exact 3D boundary representation in finite element analysis based on Cartesian grids independent of the geometry. International Journal for Numerical Methods in Engineering, 103(6):445-468, aug 2015.

[63] Toufik Kanit, Franck N'Guyen, Samuel Forest, Dominique Jeulin, Matt Reed, and Scott Singleton. Apparent and effective physical properties of heterogeneous materials: Representativity of samples of two materials from food industry. Computer Methods in Applied Mechanics and Engineering, 195(33-36):3960-3982, 2006.

[64] M. Kleiber and T.D. Hien. The stochastic finite element method (basic perturbation technique and computer implementation). Applied Stochastic Models and Data Analysis, 10(4):297-297, 1994.

[65] G. Falsone and N. Impollonia. A new approach for the stochastic analysis of finite element modelled structures with uncertain parameters. Computer Methods in Applied Mechanics and Engineering, 191(44):5067-5085, oct 2002.

[66] Dongbin Xiu. Fast Numerical Methods for Stochastic Computations: A Review. Communications in computational physics, 5(2-4):242-272, 2009.

[67] Anthony Nouy. Recent developments in spectral stochastic methods for the numerical solution of stochastic partial differential equations. Archives of Computational Methods in Engineering, 16(3):251-285, may 2009. 
[68] Manas K. Deb, Ivo Babuška, and J. Tinsley Oden. Solution of stochastic partial differential equations using Galerkin finite element techniques. Computer Methods in Applied Mechanics and Engineering, 190(48):6359-6372, 2001.

[69] O. P. Le Matîre, O. M. Knio, H. N. Najm, and R. G. Ghanem. Uncertainty propagation using Wiener-Haar expansions. Journal of Computational Physics, 197(1):2857, 2004.

[70] Xiaoliang Wan and George Em Karniadakis. An adaptive multi-element generalized polynomial chaos method for stochastic differential equations. Journal of Computational Physics, 209(2):617-642, 2005.

[71] H. Lamari, Amine Ammar, Patrice Cartraud, Grégory Legrain, Francisco Chinesta, and F. Jacquemin. Routes for Efficient Computational Homogenization of Nonlinear Materials Using the Proper Generalized Decompositions. Archives of Computational Methods in Engineering, 17(4):373-391, oct 2010.

[72] L. Giraldi, Anthony Nouy, Grégory Legrain, and P. Cartraud. Tensor-based methods for numerical homogenization from high-resolution images. Computer Methods in Applied Mechanics and Engineering, 254:154-169, 2013.

[73] Anthony Nouy. Generalized spectral decomposition method for solving stochastic finite element equations: Invariant subspace problem and dedicated algorithms. Computer Methods in Applied Mechanics and Engineering, 197(51-52):4718-4736, oct 2008 .

[74] Mark Ainsworth and J.Tinsley Tinsley Oden. A posteriori error estimation in finite element analysis. Comp. Meth. in Applied Mech. and Engrg., 7825(96):1-88, 1997.

[75] Ivo Babuška, R Tempone, and Ge Zouraris. Galerkin finite element approximations of stochastic elliptic partial differential equations. SIAM Journal on Numerical Analysis, 42(2):800-825, 2004.

[76] a J Ladd, J H Kinney, D L Haupt, and S a Goldstein. Finite-element modeling of trabecular bone: comparison with mechanical testing and determination of tissue modulus. Journal of orthopaedic research : official publication of the Orthopaedic Research Society, 16(5):622-8, sep 1998.

[77] Benedikt Helgason, Egon Perilli, Enrico Schileo, Fulvia Taddei, Sigurdur Brynjólfsson, and Marco Viceconti. Mathematical relationships between bone density and mechanical properties: a literature review. Clinical biomechanics (Bristol, Avon), 23(2):135-46, feb 2008. 